\title{
GLOBULAR AND OPEN CLUSTERS OBSERVED BY SDSS/SEGUE: THE GIANT STARS
}

\author{
Heather L. Morrison ${ }^{1}$, Zhibo Ma ${ }^{1}$, James L. Clem ${ }^{2}$, Deokkeun An ${ }^{3}$, Thomas Connor ${ }^{1,10}$, Andrew Schechtman-Rook ${ }^{1,11}$, \\ Luca Casagrande ${ }^{4}$, Constance Rockosi ${ }^{5}$, Brian Yanny ${ }^{6}$, Paul Harding ${ }^{1}$, Timothy C. Beers ${ }^{7}$, \\ JENNifer A. JOHNSON ${ }^{8}$, AND DONALd P. SCHNEIDER 9 \\ ${ }^{1}$ Department of Astronomy, Case Western Reserve University, Cleveland, OH 44106, USA; hlm5@ case.edu \\ ${ }^{2}$ Department of Physics, Grove City College, 100 Campus Dr., Grove City, PA 16127, USA \\ ${ }^{3}$ Department of Science Education, Ewha Womans University, Seoul 120-750, Korea \\ ${ }^{4}$ Research School of Astronomy and Astrophysics, Mount Stromlo Observatory, The Australian National University, ACT 2611, Australia \\ ${ }^{5}$ UCO/Lick Observatory, University of California, Santa Cruz, 1156 High St., Santa Cruz, CA 95064, USA \\ ${ }^{6}$ Fermi National Accelerator Laboratory, P.O. Box 500, Batavia IL 60510, USA \\ ${ }^{7}$ Department of Physics, University of Notre Dame, 225 Nieuwland Science Hall, Notre Dame, IN 46656, USA \\ ${ }^{8}$ Department of Astronomy, Ohio State University, 140 West 18th Avenue, Columbus, OH 43210, USA \\ ${ }^{9}$ Department of Astronomy and Astrophysics, The Pennsylvania State University, University Park, PA 16802, USA \\ Received 2014 August 19; accepted 2015 October 14; published 2015 December 21
}

\begin{abstract}
We present griz observations for the clusters M92, M13 and NGC 6791 and gr photometry for M71, Be 29 and NGC 7789. In addition we present new membership identifications for all these clusters, which have been observed spectroscopically as calibrators for the Sloan Digital Sky Survey (SDSS)/SEGUE survey; this paper focuses in particular on the red giant branch stars in the clusters. In a number of cases, these giants were too bright to be observed in the normal SDSS survey operations, and we describe the procedure used to obtain spectra for these stars. For M71, we also present a new variable reddening map and a new fiducial for the $g r$ giant branch. For NGC 7789, we derived a transformation from $T_{\text {eff }}$ to $g-r$ for giants of near solar abundance, using IRFM $T_{\text {eff }}$ measures of stars with good ugriz and 2MASS photometry and SEGUE spectra. The result of our analysis is a robust list of known cluster members with correctly dereddened and (if needed) transformed $g r$ photometry for crucial calibration efforts for SDSS and SEGUE.
\end{abstract}

Key words: globular clusters: general - open clusters and associations: general

\section{INTRODUCTION}

Calibrations which relate observables such as stellar photometry and spectroscopy to fundamental stellar parameters are a vital part of any survey. The Sloan Digital Sky Survey (SDSS: York et al. 2000) provided imaging in five passbands (ugriz, ) for 14,555 square degrees of the sky, using a dedicated imager (Gunn et al. 1998) on the SDSS $2.5 \mathrm{~m}$ telescope (Gunn et al. 2006). This photometric database was complemented by spectroscopic observations using a multi-object spectrograph (Smee et al. 2013). The original purpose of the SDSS survey was to map the extragalactic universe by obtaining spectra of one million galaxies and one hundred thousand quasars. However, because of a number of important, serendipitous discoveries on the Milky Way, two surveys (SEGUE-1 and -2) which focused on the stellar populations in the Milky Way were carried out as extensions to the original SDSS. SEGUE-1 (Yanny et al. 2009) acquired data from 2005 through 2008, and SEGUE-2 (Eisenstein et al. 2011; C. Rockosi et al. 2015, in preparation) in 2008 and 2009.

Because the SDSS ugriz photometric system (Fukugita et al. 1996) was originally designed for the study of galaxies and quasars rather than stars (focusing on avoiding strong sky lines rather than on features in a zero redshift stellar spectrum), one of the important tasks for the SEGUE survey was to obtain observations in ugriz for well-studied objects with known stellar parameters. This program has allowed us to understand

\footnotetext{
${ }^{10}$ Present address: Department of Physics and Astronomy, Michigan State University.

${ }^{11}$ Current address: Department of Astronomy, University of Wisconsin, 475 North Charter Street, Madison, WI 53706.
}

how $T_{\text {eff }}, \log g$ and $[\mathrm{Fe} / \mathrm{H}]$ map into the SDSS colors. Such understanding is particularly important when studying stars from minority populations in the Galaxy such as its halo, as there are often $[\mathrm{Fe} / \mathrm{H}]$ and luminosity terms in transformations from other photometric systems to ugriz, particularly in the $u$ and $g$ filters (for example, see Figure 10 of Yanny et al. 2009). Open and globular clusters are particularly suitable as calibrators because they provide many objects with the same values of $[\mathrm{Fe} / \mathrm{H}]$.

The first order of business in using star clusters is to obtain a good color-magnitude diagram (CMD) in the appropriate filter set. Because the SDSS photometric pipeline (Stoughton et al. 2002) does not perform well in crowded fields, An et al. (2008) performed DAOPHOT photometry of open and globular clusters imaged by SDSS. An et al. (2008) then provided accurate fiducial sequences for 17 globular and 3 open clusters covering a metallicity range from $[\mathrm{Fe} / \mathrm{H}]=-2.4$ to +0.4 . However, since the SDSS camera saturates at around $g=14.5$, and a number of these clusters have giant branches reaching significantly brighter than this limit, it was necessary to use observations from other telescopes. The $u^{\prime} g^{\prime} r^{\prime} i^{\prime} z^{\prime}$ system is defined by the same filters as the ugriz system and was intended to simplify observations in ugriz from other telescopes (Smith et al. 2002; Tucker et al. 2006). However, because the filters are in vacuum in the SDSS imager but not when used in other telescopes, the two photometric systems are in fact different. We used the $u^{\prime} g^{\prime} r^{\prime} i^{\prime} z^{\prime}$ observations of the bright giant branches of these clusters (Clem et al. 2008), transforming them to ugriz using the transformations of Tucker et al. (2006). 
The next step in the use of clusters as calibrators is to make sure that the stars we study are in fact cluster members. Spectroscopic observations provide velocities and other useful discriminants of membership, and the SEGUE survey obtained spectra of stars in 13 clusters (Lee et al. 2008b; Smolinski et al. 2011). These two papers used velocity, position on the CMD and the metallicity of the star as measured by the SEGUE Stellar Parameters Pipeline (SSPP: Allende Prieto et al. 2008; Lee et al. 2008a, 2008b; Smolinski et al. 2011) as membership criteria.

Our particular interest is the cluster red giant branches. For technical reasons described below, the giant branch stars were not well covered in the previous tests of the SSPP by Lee et al. (2008b) and Smolinski et al. (2011). The next paper in this series (H. Morrison et al. 2015, in preparation) shows how we used the clusters described in this paper to test the values of $[\mathrm{Fe} / \mathrm{H}]$ and $\log g$ for cluster members, and adds an additional luminosity discriminant (the $\mathrm{Mg}$ index) to enhance the SSPP's ability to identify red giant stars. A number of the clusters described here either have low radial velocities or are located at low galactic latitude, making it difficult to distinguish cluster members from foreground disk stars using only velocity. We have chosen to identify cluster members using a different set of criteria than Lee et al. (2008b) and Smolinski et al. (2011): while we both use the SEGUE velocities and the position on the cluster CMD, we have chosen not to use the SSPP metallicity, and have added another powerful discriminant: the stellar proper motion. Proper motion data are available for all but one clusters we study. In one particularly recalcitrant case, Berkeley 29, where proper motions were not available, we used the velocity and CMD criteria, then rejected foreground dwarfs by visual inspection of the spectra.

M71 is a particularly important and difficult case. It is the only well-studied, nearby cluster with an intermediate metallicity which is accessible from the Northern Hemisphere. Unfortunately, it also has variable reddening across its face. Thus we needed to derive individual reddening estimates for different regions of the cluster (using the photometry of Clem et al. 2008) before we could produce a cluster CMD suitable for producing a fiducial for the giant branch.

In this paper we present, for each cluster, a CMD showing the stars which are likely members and were observed spectroscopically by SEGUE, and a table of accurate coordinates and other information on these likely cluster members. We also describe the SEGUE observations of cluster stars when the cluster focal plane fiber plug-plates (plates hereafter) differed in observational procedure from the usual survey plates. In addition we provide a table of our derived spatial reddening offsets for M71 and a transformation between $T_{\text {eff }}$ and $g-r$ for near solar abundance giants.

\section{CLUSTERS USED FOR CALIBRATION}

The SEGUE project observed a number of globular and open clusters for calibration purposes. For calibration of the red giants, we selected the globular clusters M92, M13 and M71 (spanning metallicities from -2.4 to -0.8 ) and the open clusters Be 29, NGC 7789 and NGC 6791, whose [Fe/H] values range from -0.4 to +0.4 . In all but one case, the clusters are within the SDSS footprint and so ugriz photometry is available for the cluster stars.

The SDSS cluster images were analyzed using DAOPHOT (Stetson 1987) by An et al. (2008) because the SDSS photometric pipeline was not designed to handle crowded fields. However, in most cases the cluster giant branch stars were too bright to be observed in the standard ugriz system (defined by the data taken on the SDSS $2.5 \mathrm{~m}$ ) because they are saturated in the SDSS exposures. In these cases we used the $u^{\prime}$ $g^{\prime} \quad r^{\prime} i^{\prime} z^{\prime}$ photometry described in Clem et al. (2008), transforming using the equations of Tucker et al. (2006). An et al. (2008) checked these transformations using data available in both ugriz and $u^{\prime} g^{\prime} r^{\prime} i^{\prime} z^{\prime}$ and found agreement at better than the $2 \%$ level for all clusters except NGC 6791, where stars at the tip of the giant branch were redder in standard ugriz than in transformed $u^{\prime} g^{\prime} r^{\prime} i^{\prime} z^{\prime}$ by $0.05-0.10 \mathrm{mag}$. This discrepancy becomes particularly significant redder than $g-r=1.0$. Fortunately, NGC 6791 is sufficiently distant that we are able to use the SDSS ugriz for all its red giants.

We summarize the values of distance modulus, $[\mathrm{Fe} / \mathrm{H}]$ and $E(B-V)$ that we adopted for these clusters, along with the sources of these measurements, in Table 1. For the globular clusters we use the metallicity scale of Kraft \& Ivans (2003), based on Fe II lines.

In addition, we checked the list of globular cluster variables compiled by Christine Clement ${ }^{12}$ to see if any of the stars that we observed were long-period variables, since their use as calibrators would be unwise. We found that there were no known LPVs in our sample of globular cluster stars.

The SEGUE-1 survey is described in Yanny et al. (2009). The survey obtained low-resolution $(R \sim 1800)$ spectra for the wavelength region from 3800 to $9000 \AA$. Each spectroscopic pointing had a bright and faint plug-plate (plate hereafter), with exposure times of typically one and two hours respectively. This procedure allowed us to reduce the effect of scattered light from bright stars in adjacent fibers to fainter stars by limiting the magnitude range on a given plate. For the clusters, we used a more flexible setup to attempt to obtain spectra with good signal-to-noise ratio $(\mathrm{S} / \mathrm{N})$ for as many stars as possible.

For many clusters, more than one plate was designed and observed. Table 2 summarizes the information on the plates taken for each cluster discussed in this paper; often a cluster had both a "bright" and a "faint" plate designed. For the brightest stars in M92 and M13, only a very short exposure, of duration 1-2 minutes, was needed. This caused a possible problem with our spectroscopic reduction pipeline, since it uses night sky lines in the spectra to check the wavelength calibration, and such short exposures are too short to properly expose the sky lines. We evolved the following procedure in order to make such observations process correctly in the pipeline. The brightest stars were observed by drilling their fiber holes on the bright plate at a position offset by 0.02 in $\mathrm{R}$. A. $(\cos (\text { decl. }))^{-1}$. This offset is $\sim 1.5$ arcmin for M92 and M13. While the rest of the stars on the bright plate were observed, a sky spectrum accumulated in these fibers. When the "bright" exposure was finished, the telescope was moved by this offset, taking the regular stars away from their fibers and placing the brightest stars on the fibers which had been accumulating sky photons. The plate was then exposed for an additional short time. The coordinates in the SDSS database have been corrected for these offsets. The referee has asked whether starlight could have accumulated in these offset fibers during the "sky" exposures. We note that we avoided placing fibers on the bright central regions of both clusters, with the nearest star

\footnotetext{
${ }^{12}$ Found at http://www.astro.utoronto.ca/ cclement/read.html
} 
Table 1

Cluster Properties

\begin{tabular}{llccccc}
\hline \hline ID & Alternate name & $l\left(^{\circ}\right)^{\mathrm{a}}$ & $b\left(^{\circ}\right)^{\mathrm{a}}$ & $E(B-V)$ & $(m-M)_{0}$ \\
\hline M92 & NGC 6341 & 68.34 & +34.86 & $0.02^{\mathrm{b}}$ & $14.64^{\mathrm{c}}$ & $14.38^{\mathrm{c}}$ \\
M13 & NGC 6205 & 59.01 & +40.91 & $0.02^{\mathrm{b}}$ & $-2.38^{\mathrm{d}}$ \\
M71 & NGC 6838 & 56.74 & -4.56 & $0.28^{\mathrm{e}}$ & $-1.60^{\mathrm{d}}$ \\
Be 29 & & 197.95 & +7.98 & $0.08^{\mathrm{f}}$ & $12.86^{\mathrm{e}}$ & $15.60^{\mathrm{g}}$ \\
NGC 7789 & 115.48 & -5.37 & $0.25^{\mathrm{i}}$ & $-0.81^{\mathrm{d}}$ \\
NGC 6791 & & 69.96 & $0.16^{1}$ & $-0.38^{\mathrm{h}}$ \\
\hline
\end{tabular}

Notes.

a The coordinates are based on the compilation of An et al. (2008) except for NGC 7789 and Berkeley 29.

b Harris (1996).

${ }^{c}$ Carretta et al. (2000); $(m-M)_{0}$ derived from Hipparcos (Perryman et al. 1997) subdwarf fitting.

${ }^{\mathrm{d}}$ Kraft \& Ivans (2003); their globular cluster metallicity scale is based on the FeII lines from high-resolution spectra of giants.

e Grundahl et al. (2002); $(m-M)_{0}$ derived from Hipparcos subdwarf fitting.

${ }^{\mathrm{f}}$ Carraro et al. (2004)

g Bragaglia et al. (2006).

${ }^{\mathrm{h}}[\mathrm{Fe} / \mathrm{H}]$ is the average of the Carraro et al. (2004) and Sestito et al. (2008) values.

${ }^{\mathrm{i}}$ Bartašiutė \& Tautvaišienè (2004)

j Tautvaišienè et al. (2005).

k Jacobson et al. (2011).

${ }^{1}$ Brogaard (2011); $(m-M)_{0}$ is based on $(m-M)_{V}$ assuming $A_{V}=3.1 * E(B-V)$.

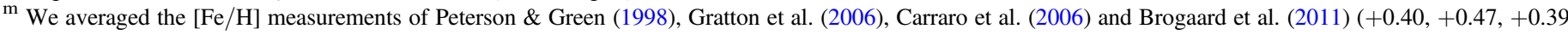
and +0.29 respectively).

Table 2

Plate Information for Clusters in this Paper

\begin{tabular}{lllll}
\hline \hline Cluster ID & Alternate & Plate & MJD & Plate type \\
\hline M92 & NGC 6341 & 2247 & 53857 & Offset \\
M92 & NGC 6341 & 2247 & 54169 & Bright \\
M92 & NGC 6341 & 2256 & 53859 & Faint \\
M13 & NGC 6205 & 2255 & 53565 & Offset \\
M13 & NGC 6205 & 2255 & 53565 & Very Bright \\
M13 & NGC 6205 & 2174 & 53521 & Bright \\
M13 & NGC 6205 & 2185 & 53532 & Faint \\
M71 & NGC 6838 & 2333 & 53682 & $\ldots$ \\
M71 & NGC 6838 & 2338 & 53679 & $\ldots$ \\
Be 29 & $\ldots$ & 3334 & 54927 & $\ldots$ \\
Be 29 & $\ldots$ & 3335 & 54922 & $\ldots$ \\
NGC 7789 & $\ldots$ & 2337 & 53991 & Bright \\
NGC 6791 & $\ldots$ & 2800 & 54326 & Bright \\
NGC 6791 & $\ldots$ & 2821 & 54393 & Faint \\
\hline
\end{tabular}

more than $2^{\prime}$ from the cluster center in M92 and $\sim 1^{\prime}$ from the less crowded center of M13. Thus it is unlikely that any light from cluster stars will contaminate the M92 offset fibers, and a little more likely for M13, where we note that in some of the stars observed with offsets, the $\mathrm{S} / \mathrm{N}$ is lower than expected at the blue end of the spectrum (4000 $\AA$ and below).

In addition, this offset procedure may introduce additional uncertainties on the radial velocities. Yanny et al. (2009) quantify the radial velocity uncertainty for SEGUE survey plates as a function of both $g$ magnitude and $\mathrm{S} / \mathrm{N}$. The CMDs of the clusters in the following section show that SEGUE observed stars as faint as $g=20$, with a wide range of $g-r$ color. At magnitudes greater than $g=19$, velocity errors can be $20 \mathrm{~km} \mathrm{~s}^{-1}$ or more. However, the red giants in the clusters, the major object of this paper, are redder and in general much brighter. For all clusters except Be 29, the giants have $g<16$, which give radial velocity errors around $5 \mathrm{~km} \mathrm{~s}^{-1}$ for the red giants. Be 29's giants can be as faint as 19, and so we expect

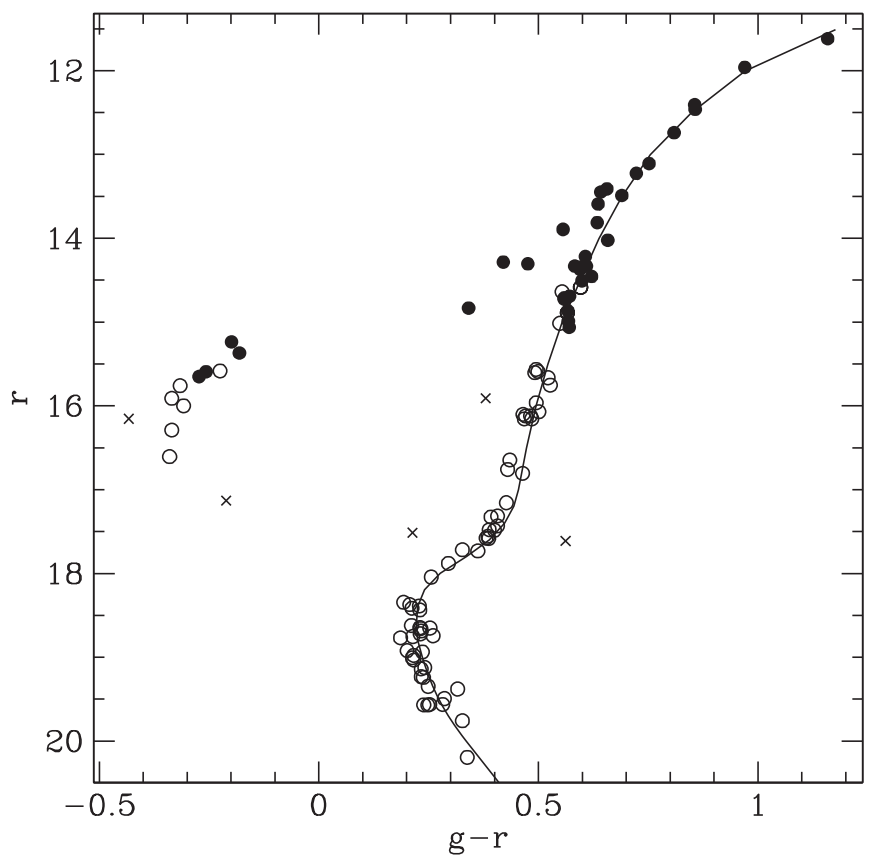

Figure 1. $g r$ CMD of the globular cluster M92, using data from An et al. (2008) and Clem et al. (2008). All points plotted are radial velocity members. Stars with proper motion membership probabilities higher than $70 \%$ are shown as filled circles, while stars with no proper motions available are shown with open circles. Crosses are stars which are classified as non-members because of their position in the CMD. The solid line is the fiducial of Clem et al. (2008), transformed to $g r$ using the transformation of Tucker et al. (2006).

larger velocity errors for this cluster of up to $10 \mathrm{~km} \mathrm{~s}^{-1}$. The two clusters with offset observations (M92 and M13) may have an additional error of a few $\mathrm{km} \mathrm{s}^{-1}$ introduced for the brightest stars.

The spectroscopic observations of the near solar metallicity open cluster NGC 7789 were targeted on the giant branch only, 
Table 3

M92 Members Observed by SEGUE

\begin{tabular}{|c|c|c|c|c|c|c|c|c|c|c|c|c|}
\hline Plate & MJD & Fiber & $\begin{array}{c}r \\
(\mathrm{mag})\end{array}$ & $\begin{array}{l}\text { Error } \\
\text { (mag) }\end{array}$ & $\begin{array}{c}g-r \\
(\mathrm{mag})\end{array}$ & $\begin{array}{c}g-i \\
(\mathrm{mag})\end{array}$ & $\begin{array}{c}g-z \\
(\mathrm{mag})\end{array}$ & $\begin{array}{l}\text { R.A. } \\
\text { (2000) }\end{array}$ & $\begin{array}{l}\text { Decl. } \\
(2000)\end{array}$ & $\begin{array}{l}\text { Velocity } \\
\left(\mathrm{km} \mathrm{s}^{-1}\right)\end{array}$ & $\begin{array}{c}\text { Phot. } \\
\text { References }\end{array}$ & $\begin{array}{c}\text { PM prob } \\
(\%)\end{array}$ \\
\hline 2247 & 53857 & 402 & 14.456 & 0.006 & 0.621 & 0.893 & 1.040 & 259.1997 & 43.1044 & -114.6 & 1 & 99 \\
\hline 2247 & 53857 & 403 & 14.218 & 0.006 & 0.607 & 0.884 & 1.047 & 259.1831 & 43.1255 & -103.4 & 1 & 99 \\
\hline 2247 & 53857 & 412 & 13.410 & 0.002 & 0.656 & 0.963 & 1.148 & 259.1429 & 43.1268 & -113.3 & 1 & 99 \\
\hline 2247 & 53857 & 416 & 13.448 & 0.003 & 0.642 & 0.948 & 1.128 & 259.1572 & 43.1448 & -122.2 & 1 & 99 \\
\hline 2247 & 53857 & 444 & 13.592 & 0.003 & 0.636 & 0.943 & 1.116 & 259.2514 & 43.1966 & -109.5 & 1 & 99 \\
\hline 2247 & 53857 & 449 & 13.226 & 0.002 & 0.723 & 1.065 & 1.234 & 259.2452 & 43.2532 & -107.9 & 1 & 99 \\
\hline 2247 & 53857 & 453 & 14.333 & 0.038 & 0.583 & 0.842 & 0.976 & 259.2280 & 43.1740 & -110.0 & 1 & 99 \\
\hline 2247 & 53857 & 455 & 12.462 & 0.004 & 0.857 & 1.252 & 1.454 & 259.2078 & 43.1781 & -115.4 & 1 & 99 \\
\hline 2247 & 53857 & 458 & 13.109 & 0.002 & 0.752 & 1.107 & 1.276 & 259.2406 & 43.2365 & -118.0 & 1 & 99 \\
\hline 2247 & 53857 & 460 & 13.813 & 0.011 & 0.634 & 0.969 & 1.142 & 259.2313 & 43.0843 & -120.1 & 1 & 99 \\
\hline 2247 & 53857 & 486 & 14.586 & 0.005 & 0.596 & 0.865 & 1.027 & 259.1491 & 42.9443 & -118.5 & 1 & $\ldots$ \\
\hline 2247 & 53857 & 514 & 14.344 & 0.003 & 0.596 & 0.877 & 1.028 & 259.2487 & 43.0183 & -119.8 & 1 & 99 \\
\hline 2247 & 53857 & 516 & 14.285 & 0.005 & 0.420 & 0.614 & 0.684 & 259.2202 & 43.0582 & -110.1 & 1 & 99 \\
\hline 2247 & 53857 & 522 & 11.619 & 0.006 & 1.159 & 1.659 & 1.930 & 259.3405 & 43.2149 & -103.4 & 1 & 99 \\
\hline 2247 & 53857 & 523 & 12.740 & 0.003 & 0.809 & 1.176 & 1.360 & 259.3189 & 43.1792 & -104.6 & 1 & 99 \\
\hline 2247 & 53857 & 525 & 14.024 & 0.014 & 0.658 & 0.933 & 1.095 & 259.3403 & 43.1842 & -110.7 & 1 & 99 \\
\hline 2247 & 53857 & 526 & 11.961 & 0.011 & 0.970 & 1.441 & 1.702 & 259.2935 & 43.1855 & -106.2 & 1 & 99 \\
\hline 2247 & 53857 & 529 & 13.895 & 0.005 & 0.556 & 0.824 & 0.980 & 259.3320 & 43.2451 & -112.3 & 1 & 99 \\
\hline 2247 & 53857 & 532 & 14.305 & 0.009 & 0.476 & 0.691 & 0.818 & 259.3376 & 43.1035 & -108.7 & 1 & 99 \\
\hline 2247 & 53857 & 537 & 14.332 & 0.006 & 0.609 & 0.887 & 1.032 & 259.3146 & 43.0831 & -113.3 & 1 & 99 \\
\hline 2247 & 53857 & 553 & 14.833 & 0.001 & 0.341 & 0.486 & 0.583 & 259.3048 & 43.0001 & -102.9 & 1 & 99 \\
\hline 2247 & 53857 & 559 & 14.371 & 0.005 & 0.595 & 0.875 & 1.033 & 259.2664 & 43.0341 & -110.2 & 1 & 99 \\
\hline 2247 & 53857 & 572 & 12.407 & 0.003 & 0.856 & 1.230 & 1.459 & 259.3821 & 43.0949 & -107.3 & 1 & 99 \\
\hline 2247 & 53857 & 576 & 14.507 & 0.010 & 0.599 & 0.879 & 1.035 & 259.3699 & 43.1675 & -114.0 & 1 & 99 \\
\hline 2247 & 53857 & 577 & 14.691 & 0.004 & 0.571 & 0.831 & 0.982 & 259.3709 & 43.1153 & -114.1 & 1 & 99 \\
\hline 2247 & 53857 & 580 & 14.353 & 0.009 & 0.598 & 0.883 & 1.020 & 259.3730 & 43.2041 & -113.7 & 1 & 99 \\
\hline 2247 & 53857 & 583 & 13.489 & 0.005 & 0.690 & 1.004 & 1.200 & 259.3425 & 43.0805 & -112.8 & 1 & 99 \\
\hline 2247 & 53857 & 609 & 14.660 & 0.005 & 0.606 & 0.871 & 0.998 & 259.5197 & 43.1712 & -118.5 & 2 & 84 \\
\hline 2247 & 53857 & 612 & 14.889 & 0.002 & 0.566 & 0.832 & 0.977 & 259.4598 & 43.2295 & -105.3 & 1 & $\ldots$ \\
\hline 2247 & 54169 & 361 & 15.583 & 0.004 & -0.225 & -0.395 & -0.463 & 259.0527 & 43.1739 & -115.1 & 1 & $\ldots$ \\
\hline 2247 & 54169 & 380 & 15.664 & 0.002 & 0.522 & 0.749 & 0.904 & 259.1245 & 43.1009 & -109.5 & 1 & $\ldots$ \\
\hline 2247 & 54169 & 408 & 14.865 & 0.007 & 0.567 & 0.816 & 0.973 & 259.1516 & 43.1156 & -120.0 & 1 & 99 \\
\hline 2247 & 54169 & 418 & 15.063 & 0.003 & 0.570 & 0.810 & 0.959 & 259.1925 & 43.0829 & -119.6 & 1 & 99 \\
\hline 2247 & 54169 & 441 & 15.760 & 0.002 & -0.316 & -0.533 & -0.641 & 259.2120 & 43.1897 & -115.4 & 1 & $\ldots$ \\
\hline 2247 & 54169 & 444 & 15.590 & 0.003 & 0.499 & 0.740 & 0.871 & 259.1783 & 43.2465 & -112.3 & 1 & $\ldots$ \\
\hline 2247 & 54169 & 449 & 15.015 & 0.003 & 0.549 & 0.800 & 0.946 & 259.2012 & 43.1713 & -116.5 & 1 & $\ldots$ \\
\hline 2247 & 54169 & 451 & 15.753 & 0.003 & 0.527 & 0.744 & 0.888 & 259.2681 & 43.0696 & -121.9 & 1 & $\ldots$ \\
\hline 2247 & 54169 & 452 & 15.565 & 0.005 & 0.495 & 0.746 & 0.897 & 259.1898 & 43.2296 & -121.4 & 1 & $\begin{array}{l}\cdots \\
\ldots\end{array}$ \\
\hline 2247 & 54169 & 484 & 14.586 & 0.005 & 0.596 & 0.865 & 1.027 & 259.1490 & 42.9443 & -115.0 & 1 & $\ldots$ \\
\hline 2247 & 54169 & 504 & 14.638 & 0.022 & 0.554 & 0.839 & 1.002 & 259.3471 & 42.9488 & -112.1 & 1 & $\ldots$ \\
\hline 2247 & 54169 & 521 & 15.651 & 0.004 & -0.273 & -0.454 & -0.545 & 259.2528 & 43.2175 & -119.3 & 1 & 99 \\
\hline 2247 & 54169 & 526 & 15.592 & 0.004 & -0.257 & -0.447 & -0.527 & 259.2901 & 43.0796 & -117.6 & 1 & 99 \\
\hline 2247 & 54169 & 531 & 16.043 & 0.005 & 0.511 & 0.724 & 0.830 & 259.3130 & 43.2645 & -115.2 & 2 & $\ldots$ \\
\hline 2247 & 54169 & 538 & 17.658 & 0.007 & 0.373 & 0.525 & 0.583 & 259.3413 & 43.2580 & -107.6 & 2 & $\begin{array}{l}\cdots \\
\cdots\end{array}$ \\
\hline 2247 & 54169 & 549 & 15.237 & 0.006 & -0.199 & -0.343 & -0.381 & 259.4017 & 43.0199 & -121.2 & 1 & 97 \\
\hline 2247 & 54169 & 563 & 14.989 & 0.006 & 0.568 & 0.816 & 0.955 & 259.3296 & 43.2152 & -111.7 & 1 & 99 \\
\hline 2247 & 54169 & 565 & 15.910 & 0.008 & -0.335 & -0.579 & -0.701 & 259.3446 & 43.1587 & -109.7 & 1 & $\ldots$ \\
\hline 2247 & 54169 & 567 & 17.255 & 0.005 & 0.453 & 0.595 & 0.626 & 259.4499 & 43.3070 & -118.4 & 2 & $\ldots$ \\
\hline 2247 & 54169 & 568 & 15.370 & 0.006 & -0.181 & -0.314 & -0.357 & 259.3660 & 43.1475 & -113.8 & 1 & 99 \\
\hline 2247 & 54169 & 573 & 15.604 & 0.002 & 0.492 & 0.689 & 0.796 & 259.3214 & 43.0742 & -116.3 & 1 & $\ldots$ \\
\hline 2247 & 54169 & 575 & 17.519 & 0.006 & 0.427 & 0.587 & 0.654 & 259.3812 & 43.2469 & -111.4 & 2 & $\begin{array}{l}\cdots \\
\cdots\end{array}$ \\
\hline 2247 & 54169 & 581 & 15.963 & 0.007 & 0.495 & 0.711 & 0.837 & 259.3938 & 43.0711 & -110.5 & 1 & $\ldots$ \\
\hline 2247 & 54169 & 584 & 17.419 & 0.006 & 0.434 & 0.615 & 0.704 & 259.4844 & 43.0595 & -103.4 & 2 & $\ldots$ \\
\hline 2247 & 54169 & 589 & 16.587 & 0.006 & 0.481 & 0.675 & 0.802 & 259.4322 & 43.0634 & -114.7 & 2 & $\ldots$ \\
\hline 2247 & 54169 & 601 & 16.062 & 0.005 & 0.517 & 0.729 & 0.817 & 259.5772 & 43.1990 & -112.1 & 2 & $\ldots$ \\
\hline 2247 & 54169 & 608 & 14.889 & 0.002 & 0.566 & 0.832 & 0.977 & 259.4598 & 43.2295 & -120.3 & 1 & $\ldots$ \\
\hline 2247 & 54169 & 610 & 14.660 & 0.005 & 0.606 & 0.871 & 0.998 & 259.5197 & 43.1712 & -117.2 & 2 & $\ldots$ \\
\hline 2247 & 54169 & 612 & 15.999 & 0.007 & -0.308 & -0.527 & -0.636 & 259.4042 & 43.1310 & -116.4 & 1 & $\ldots$ \\
\hline 2247 & 54169 & 616 & 17.522 & 0.005 & 0.433 & 0.598 & 0.668 & 259.3905 & 43.1896 & -115.4 & 2 & $\ldots$ \\
\hline 2247 & 54169 & 620 & 17.097 & 0.005 & 0.473 & 0.663 & 0.741 & 259.4374 & 43.1356 & -103.9 & 2 & $\ldots$ \\
\hline 2256 & 53859 & 521 & 18.947 & 0.012 & 0.259 & 0.361 & 0.297 & 259.3361 & 43.2903 & -117.3 & 2 & $\ldots$ \\
\hline 2256 & 53859 & 525 & 18.285 & 0.009 & 0.239 & 0.343 & 0.406 & 259.3690 & 43.2710 & -111.2 & 2 & $\begin{array}{l}\cdots \\
\cdots\end{array}$ \\
\hline 2256 & 53859 & 534 & 19.172 & 0.014 & 0.279 & 0.337 & 0.335 & 259.3679 & 43.2307 & -104.4 & 2 & $\ldots$ \\
\hline
\end{tabular}


Table 3

(Continued)

\begin{tabular}{|c|c|c|c|c|c|c|c|c|c|c|c|c|}
\hline Plate & MJD & Fiber & $\begin{array}{c}r \\
(\mathrm{mag})\end{array}$ & $\begin{array}{l}\text { Error } \\
(\mathrm{mag})\end{array}$ & $\begin{array}{c}g-r \\
(\mathrm{mag})\end{array}$ & $\begin{array}{c}g-i \\
(\mathrm{mag})\end{array}$ & $\begin{array}{c}g-z \\
(\mathrm{mag})\end{array}$ & $\begin{array}{l}\text { R.A. } \\
(2000)\end{array}$ & $\begin{array}{l}\text { Decl. } \\
(2000)\end{array}$ & $\begin{array}{r}\text { Velocity } \\
\left(\mathrm{km} \mathrm{s}^{-1}\right)\end{array}$ & $\begin{array}{c}\text { Phot. } \\
\text { References }\end{array}$ & $\begin{array}{c}\text { PM prob } \\
(\%)\end{array}$ \\
\hline 2256 & 53859 & 537 & 19.080 & 0.014 & 0.279 & 0.369 & 0.416 & 259.4246 & 43.1206 & -112.4 & 2 & $\ldots$ \\
\hline 2256 & 53859 & 537 & 20.197 & 0.036 & 0.525 & 0.708 & 0.760 & 259.4246 & 43.1206 & -112.4 & 2 & $\ldots$ \\
\hline 2256 & 53859 & 539 & 18.661 & 0.011 & 0.277 & 0.405 & 0.452 & 259.3406 & 43.2491 & -113.1 & 2 & $\ldots$ \\
\hline 2256 & 53859 & 561 & 18.975 & 0.013 & 0.262 & 0.359 & 0.367 & 259.4673 & 43.1504 & -112.9 & 2 & $\ldots$ \\
\hline 2256 & 53859 & 562 & 18.709 & 0.012 & 0.232 & 0.323 & 0.270 & 259.3947 & 43.2326 & -119.8 & 2 & $\ldots$ \\
\hline 2256 & 53859 & 569 & 19.511 & 0.017 & 0.294 & 0.427 & 0.480 & 259.4556 & 43.1986 & -109.2 & 2 & $\ldots$ \\
\hline 2256 & 53859 & 576 & 18.356 & 0.008 & 0.258 & 0.320 & 0.334 & 259.4356 & 43.1723 & -109.1 & 2 & $\ldots$ \\
\hline 2256 & 53859 & 578 & 19.320 & 0.021 & 0.362 & 0.458 & 0.416 & 259.4461 & 43.1315 & -103.3 & 2 & $\ldots$ \\
\hline 2256 & 53859 & 604 & 18.693 & 0.010 & 0.260 & 0.349 & 0.385 & 259.5561 & 43.2827 & -121.2 & 2 & $\ldots$ \\
\hline 2256 & 53859 & 608 & 17.984 & 0.008 & 0.302 & 0.393 & 0.419 & 259.4972 & 43.3424 & -122.2 & 2 & $\ldots$ \\
\hline 2256 & 53859 & 612 & 18.313 & 0.010 & 0.253 & 0.345 & 0.332 & 259.4838 & 43.2025 & -115.4 & 2 & $\ldots$ \\
\hline
\end{tabular}

References. 1. Clem et al. (2008), 2. An et al. (2008).

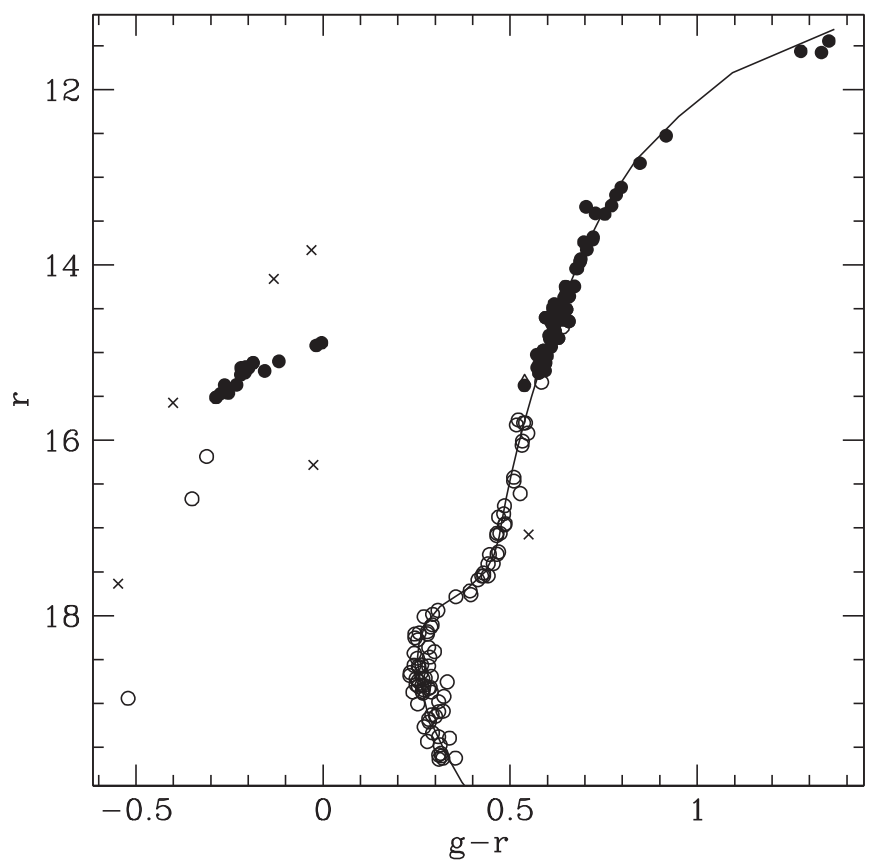

Figure 2. $g r \mathrm{CMD}$ of the globular cluster M13, using data from An et al (2008) and Clem et al. (2008). All points plotted are radial velocity members. Symbols have the same meaning as in Figure 1. The solid line shows the transformed fiducial of Clem et al. (2008).

because there are already good SEGUE spectroscopic observations of stars below the main-sequence turnoff in two open clusters with $[\mathrm{Fe} / \mathrm{H}]$ close to solar: M67 and NGC 2420.

\section{CLUSTERS WITH PROPER MOTION DATA}

We used the proper motions of Cudworth and collaborators for M92, M13, M71 (Cudworth \& Monet 1979; Cudworth 1985; Rees 1992, respectively) and for NGC 6791 (K. Cudworth 2015, private communication). For NGC 7789 we used the proper motions of McNamara \& Solomon (1981).

Because the radial velocity zeropoint was uncertain for some of the brighter cluster plates, we first examined the proper motion members (those with membership probability greater than $70 \%$ ) to obtain a clean radial velocity distribution for the cluster. We then used this to find the optimal range of radial velocities for cluster membership selection.

\section{1. $M 92$}

For the most metal-poor globular cluster in our dataset, M92, the giant branch tip is at $r \sim 11.5$, so most of the red giant branch is saturated in the SDSS photometry. We therefore used both the ugriz photometry of An et al. (2008) (reference run 4682 plus run 5237, transformed to the reference frame using the transformations given in An et al. 2008) and the transformed photometry of Clem et al. (2008) to construct the CMD (Figure 1).

SDSS photometric reductions have improved over the years of the survey. An important advance occurred between Data Releases (DR) 7 and 8: what is known as the "Ubercalibration" (Padmanabhan et al. 2008). This technique solves for the photometric calibration parameters using all overlapping observations. When An et al. (2008) first made the DAOPHOT reductions of the cluster data available, they calibrated these reductions to DR7. Subsequently, An et al. (2013) calculated the offsets to apply in order to put the cluster photometry on the DR8 system, and we have applied these offsets to the An et al. photometry given in Table 3 .

Proper motions from Rees (1992) are available for all giants above the level of the horizontal branch. We chose a radial velocity range of -123 to $-103 \mathrm{~km} \mathrm{~s}^{-1}$ for cluster members. Coordinates, griz photometry and its source, SEGUE radial velocities and proper motion membership probabilities for each member are given in Table 3.

\section{2. $M 13$}

M13 also has bright giants, so the CMD shown in Figure 2 uses photometry from both An et al. (2008) and Clem et al. (2008). For the photometry from An et al. (2008), we used runs 3225 and 3226, correcting run 3226 to the reference run (3225) using the corrections given in An et al. (2008), and then applied the "Ubercalibration" corrections given in Table 1 of An et al. 
Table 4

M13 Members Observed by SEGUE

\begin{tabular}{|c|c|c|c|c|c|c|c|c|c|c|c|c|}
\hline Plate & MJD & Fiber & $\begin{array}{c}r \\
(\mathrm{mag})\end{array}$ & $\begin{array}{l}\text { Error } \\
(\mathrm{mag})\end{array}$ & $\begin{array}{c}g-r \\
(\mathrm{mag})\end{array}$ & $\begin{array}{c}g-i \\
(\mathrm{mag})\end{array}$ & $\begin{array}{c}g-z \\
(\mathrm{mag})\end{array}$ & $\begin{array}{l}\text { R.A. } \\
\text { (2000) }\end{array}$ & $\begin{array}{l}\text { Decl. } \\
(2000)\end{array}$ & $\begin{array}{l}\text { Velocity } \\
\left(\mathrm{km} \mathrm{s}^{-1}\right)\end{array}$ & $\begin{array}{l}\text { Phot. } \\
\text { ref. }\end{array}$ & $\begin{array}{c}\text { PM prob } \\
(\%)\end{array}$ \\
\hline 2174 & 53521 & 128 & 17.060 & 0.013 & 0.471 & 0.682 & 0.753 & 250.4742 & 36.3098 & -249.0 & 2 & $\cdots$ \\
\hline 2174 & 53521 & 133 & 16.421 & 0.010 & 0.508 & 0.728 & 0.813 & 250.5125 & 36.3211 & -242.6 & 2 & \\
\hline 2174 & 53521 & 153 & 15.124 & 0.020 & -0.159 & -0.304 & -0.347 & 250.4664 & 36.4093 & -247.8 & 2 & 99 \\
\hline 2174 & 53521 & 406 & 17.522 & 0.011 & 0.430 & 0.610 & 0.678 & 250.2975 & 36.6566 & -249.8 & 2 & $\ldots$ \\
\hline 2174 & 53521 & 444 & 14.532 & 0.005 & 0.621 & 0.913 & 1.029 & 250.3637 & 36.5395 & -243.7 & 2 & 99 \\
\hline 2174 & 53521 & 445 & 17.523 & 0.010 & 0.425 & 0.600 & 0.661 & 250.3154 & 36.5818 & -247.6 & 2 & $\ldots$ \\
\hline 2174 & 53521 & 447 & 17.276 & 0.009 & 0.466 & 0.658 & 0.708 & 250.3488 & 36.6371 & -246.1 & 2 & $\ldots$ \\
\hline 2174 & 53521 & 456 & 16.942 & 0.009 & 0.486 & 0.677 & 0.761 & 250.3559 & 36.6084 & -248.2 & 2 & $\ldots$ \\
\hline 2174 & 53521 & 461 & 16.926 & 0.008 & 0.488 & 0.686 & 0.780 & 250.4162 & 36.5927 & -245.1 & 2 & $\ldots$ \\
\hline 2174 & 53521 & 480 & 16.441 & 0.005 & 0.511 & 0.735 & 0.823 & 250.3776 & 36.5606 & -244.9 & 2 & $\cdots$ \\
\hline 2174 & 53521 & 554 & 15.314 & 0.010 & 0.585 & 0.826 & 0.949 & 250.4525 & 36.7311 & -248.1 & 2 & $\ldots$ \\
\hline 2174 & 53521 & 121 & 15.767 & 0.004 & 0.522 & 0.754 & 0.867 & 250.5334 & 36.3239 & -249.4 & 1 & $\ldots$ \\
\hline 2174 & 53521 & 131 & 14.601 & 0.022 & 0.595 & 0.878 & 1.044 & 250.4894 & 36.3321 & -243.0 & 1 & 99 \\
\hline 2174 & 53521 & 136 & 15.511 & 0.004 & -0.286 & -0.468 & -0.588 & 250.4906 & 36.3635 & -242.9 & 1 & 98 \\
\hline 2174 & 53521 & 145 & 14.357 & 0.002 & 0.658 & 0.930 & 1.063 & 250.4505 & 36.3933 & -246.4 & 1 & 99 \\
\hline 2174 & 53521 & 154 & 14.475 & 0.003 & 0.636 & 0.887 & 1.056 & 250.4662 & 36.3263 & -249.9 & 1 & 99 \\
\hline 2174 & 53521 & 156 & 15.142 & 0.006 & 0.577 & 0.830 & 0.946 & 250.3522 & 36.4095 & -239.7 & 1 & 99 \\
\hline 2174 & 53521 & 157 & 15.412 & 0.004 & -0.254 & -0.440 & -0.548 & 250.4520 & 36.3018 & -247.2 & 1 & 99 \\
\hline 2174 & 53521 & 158 & 15.112 & 0.004 & 0.586 & 0.822 & 0.957 & 250.4085 & 36.3039 & -243.9 & 1 & 99 \\
\hline 2174 & 53521 & 167 & 14.255 & 0.003 & 0.654 & 0.932 & 1.089 & 250.2756 & 36.4229 & -246.5 & 1 & 99 \\
\hline 2174 & 53521 & 168 & 14.721 & 0.003 & 0.616 & 0.874 & 1.023 & 250.2608 & 36.4377 & -244.0 & 1 & 99 \\
\hline 2174 & 53521 & 171 & 14.367 & 0.003 & 0.656 & 0.935 & 1.083 & 250.3129 & 36.3983 & -247.0 & 1 & 90 \\
\hline 2174 & 53521 & 172 & 14.753 & 0.003 & 0.619 & 0.885 & 1.026 & 250.3078 & 36.4174 & -247.8 & 1 & 99 \\
\hline 2174 & 53521 & 176 & 15.232 & 0.002 & 0.577 & 0.824 & 0.919 & 250.3261 & 36.3471 & -240.3 & 1 & 99 \\
\hline 2174 & 53521 & 412 & 17.085 & 0.004 & 0.464 & 0.657 & 0.749 & 250.2389 & 36.5871 & -242.8 & 1 & $\ldots$ \\
\hline 2174 & 53521 & 414 & 17.060 & 0.003 & 0.465 & 0.672 & 0.776 & 250.2673 & 36.5864 & -241.9 & 1 & $\cdots$ \\
\hline 2174 & 53521 & 418 & 15.919 & 0.003 & 0.547 & 0.775 & 0.923 & 250.2429 & 36.7086 & -247.4 & 1 & $\begin{array}{l}\cdots \\
\cdots\end{array}$ \\
\hline 2174 & 53521 & 442 & 15.116 & 0.002 & -0.187 & -0.328 & -0.421 & 250.3339 & 36.6145 & -249.2 & 1 & 99 \\
\hline 2174 & 53521 & 449 & 14.806 & 0.002 & 0.604 & 0.865 & 1.036 & 250.3311 & 36.5077 & -244.1 & 1 & 99 \\
\hline 2174 & 53521 & 457 & 14.620 & 0.003 & 0.612 & 0.878 & 0.974 & 250.3618 & 36.4246 & -244.6 & 1 & 99 \\
\hline 2174 & 53521 & 458 & 14.511 & 0.002 & 0.632 & 0.902 & 1.060 & 250.3154 & 36.4639 & -245.9 & 1 & 99 \\
\hline 2174 & 53521 & 459 & 14.665 & 0.001 & 0.611 & 0.886 & 1.050 & 250.3160 & 36.5549 & -245.0 & 1 & 99 \\
\hline 2174 & 53521 & 460 & 14.246 & 0.002 & 0.648 & 0.932 & 1.105 & 250.3239 & 36.4916 & -238.9 & 1 & 99 \\
\hline 2174 & 53521 & 462 & 14.560 & 0.002 & 0.623 & 0.911 & 1.056 & 250.3755 & 36.5912 & -245.5 & 1 & 99 \\
\hline 2174 & 53521 & 463 & 15.115 & 0.001 & 0.595 & 0.867 & 0.999 & 250.4507 & 36.5948 & -243.8 & 1 & 99 \\
\hline 2174 & 53521 & 464 & 15.163 & 0.003 & -0.207 & -0.331 & -0.411 & 250.3978 & 36.6046 & -239.1 & 1 & 99 \\
\hline 2174 & 53521 & 466 & 14.919 & 0.002 & -0.018 & -0.059 & -0.091 & 250.4609 & 36.5551 & -241.7 & 1 & 99 \\
\hline 2174 & 53521 & 467 & 14.620 & 0.003 & 0.614 & 0.888 & 1.071 & 250.3318 & 36.6897 & -242.9 & 1 & $\ldots$ \\
\hline 2174 & 53521 & 470 & 14.258 & 0.007 & 0.649 & 0.941 & 1.100 & 250.3886 & 36.5412 & -241.2 & 1 & 99 \\
\hline 2174 & 53521 & 471 & 15.802 & 0.002 & 0.541 & 0.777 & 0.909 & 250.4051 & 36.6807 & -248.8 & 1 & $\ldots$ \\
\hline 2174 & 53521 & 472 & 14.888 & 0.004 & -0.004 & -0.047 & -0.119 & 250.4393 & 36.4306 & -243.1 & 1 & 99 \\
\hline 2174 & 53521 & 475 & 14.333 & 0.002 & 0.649 & 0.926 & 1.086 & 250.4188 & 36.5268 & -239.4 & 1 & 99 \\
\hline 2174 & 53521 & 476 & 15.207 & 0.002 & 0.593 & 0.840 & 0.968 & 250.4538 & 36.5347 & -249.7 & 1 & 99 \\
\hline 2174 & 53521 & 477 & 14.629 & 0.002 & 0.642 & 0.904 & 1.057 & 250.4330 & 36.4116 & -240.9 & 1 & 99 \\
\hline 2174 & 53521 & 486 & 15.466 & 0.003 & -0.273 & -0.477 & -0.572 & 250.4692 & 36.5147 & -245.9 & 1 & 99 \\
\hline 2174 & 53521 & 487 & 14.507 & 0.001 & 0.651 & 0.910 & 1.078 & 250.5424 & 36.6308 & -249.8 & 1 & 98 \\
\hline 2174 & 53521 & 488 & 15.197 & 0.004 & 0.589 & 0.842 & 0.956 & 250.5207 & 36.5268 & -246.1 & 1 & 99 \\
\hline 2174 & 53521 & 489 & 15.037 & 0.003 & 0.599 & 0.855 & 1.000 & 250.5569 & 36.5541 & -246.5 & 1 & 99 \\
\hline 2174 & 53521 & 491 & 15.463 & 0.002 & -0.253 & -0.440 & -0.553 & 250.5045 & 36.5626 & -249.7 & 1 & 99 \\
\hline 2174 & 53521 & 493 & 14.519 & 0.008 & 0.633 & 0.906 & 1.022 & 250.4687 & 36.4504 & -247.9 & 1 & 99 \\
\hline 2174 & 53521 & 497 & 14.836 & 0.002 & 0.629 & 0.887 & 1.037 & 250.5392 & 36.5664 & -240.3 & 1 & 99 \\
\hline 2174 & 53521 & 498 & 14.558 & 0.002 & 0.627 & 0.897 & 1.043 & 250.5455 & 36.4092 & -240.7 & 1 & 99 \\
\hline 2174 & 53521 & 499 & 14.521 & 0.001 & 0.618 & 0.883 & 1.038 & 250.5074 & 36.3896 & -242.0 & 1 & 99 \\
\hline 2174 & 53521 & 500 & 14.756 & 0.003 & 0.620 & 0.880 & 1.039 & 250.4441 & 36.5013 & -249.6 & 1 & 99 \\
\hline 2174 & 53521 & 522 & 14.977 & 0.003 & 0.589 & 0.843 & 0.980 & 250.5715 & 36.5259 & -244.9 & 1 & 99 \\
\hline 2174 & 53521 & 524 & 14.495 & 0.002 & 0.620 & 0.900 & 1.036 & 250.5826 & 36.4961 & -245.9 & 1 & 99 \\
\hline 2174 & 53521 & 529 & 16.009 & 0.006 & 0.533 & 0.748 & 0.875 & 250.6123 & 36.6507 & -245.6 & 1 & $\ldots$ \\
\hline 2174 & 53521 & 530 & 14.717 & 0.003 & 0.617 & 0.876 & 1.033 & 250.5795 & 36.6176 & -246.8 & 1 & 99 \\
\hline 2174 & 53521 & 531 & 14.674 & 0.004 & 0.620 & 0.886 & 1.020 & 250.6084 & 36.4513 & -243.9 & 1 & 99 \\
\hline 2174 & 53521 & 532 & 15.252 & 0.002 & -0.220 & -0.361 & -0.464 & 250.5847 & 36.4509 & -245.9 & 1 & 99 \\
\hline 2174 & 53521 & 542 & 14.709 & 0.003 & 0.640 & 0.890 & 1.072 & 250.4867 & 36.6975 & -248.1 & 1 & $\ldots$ \\
\hline 2185 & 53532 & 181 & 18.574 & 0.003 & 0.282 & 0.376 & 0.353 & 250.2266 & 36.2189 & -247.5 & 1 & $\begin{array}{l}\cdots \\
\cdots\end{array}$ \\
\hline 2185 & 53532 & 421 & 18.919 & 0.012 & -0.520 & -0.859 & -1.232 & 250.3562 & 36.6878 & -246.4 & 2 & $\ldots$ \\
\hline
\end{tabular}


Table 4

(Continued)

\begin{tabular}{|c|c|c|c|c|c|c|c|c|c|c|c|c|}
\hline Plate & MJD & Fiber & $\begin{array}{c}r \\
(\mathrm{mag})\end{array}$ & $\begin{array}{l}\text { Error } \\
\text { (mag) }\end{array}$ & $\begin{array}{c}g-r \\
(\mathrm{mag})\end{array}$ & $\begin{array}{c}g-i \\
(\mathrm{mag})\end{array}$ & $\begin{array}{c}g-z \\
(\mathrm{mag})\end{array}$ & $\begin{array}{l}\text { R.A. } \\
(2000)\end{array}$ & $\begin{array}{l}\text { Decl. } \\
(2000)\end{array}$ & $\begin{array}{l}\text { Velocity } \\
\left(\mathrm{km} \mathrm{s}^{-1}\right)\end{array}$ & $\begin{array}{l}\text { Phot. } \\
\text { ref. }\end{array}$ & $\begin{array}{c}\text { PM prob } \\
(\%)\end{array}$ \\
\hline 2185 & 53532 & 461 & 18.172 & 0.011 & 0.258 & 0.359 & 0.333 & 250.3284 & 36.7000 & -241.4 & 2 & $\ldots$ \\
\hline 2185 & 53532 & 462 & 18.011 & 0.003 & 0.270 & 0.391 & 0.450 & 250.2369 & 36.7179 & -248.1 & 1 & $\ldots$ \\
\hline 2185 & 53532 & 466 & 18.649 & 0.003 & 0.233 & 0.316 & 0.376 & 250.2879 & 36.7253 & -245.5 & 1 & $\ldots$ \\
\hline 2185 & 53532 & 469 & 18.783 & 0.012 & 0.256 & 0.368 & 0.403 & 250.2945 & 36.6066 & -246.2 & 2 & $\ldots$ \\
\hline 2185 & 53532 & 475 & 18.428 & 0.001 & 0.244 & 0.338 & 0.364 & 250.2399 & 36.5889 & -249.1 & 1 & $\ldots$ \\
\hline 2185 & 53532 & 476 & 18.883 & 0.002 & 0.267 & 0.376 & 0.396 & 250.2486 & 36.5748 & -244.4 & 1 & $\ldots$ \\
\hline 2185 & 53532 & 477 & 19.381 & 0.002 & 0.309 & 0.415 & 0.480 & 250.2305 & 36.6108 & -244.1 & 1 & $\ldots$ \\
\hline 2185 & 53532 & 478 & 18.732 & 0.023 & 0.333 & 0.432 & 0.452 & 250.2752 & 36.6187 & -243.6 & 2 & $\ldots$ \\
\hline 2185 & 53532 & 480 & 19.474 & 0.005 & 0.313 & 0.429 & 0.490 & 250.2232 & 36.6269 & -243.0 & 1 & $\ldots$ \\
\hline 2185 & 53532 & 481 & 17.961 & 0.012 & 0.294 & 0.422 & 0.475 & 250.3259 & 36.6554 & -244.6 & 2 & $\ldots$ \\
\hline 2185 & 53532 & 485 & 18.251 & 0.009 & 0.254 & 0.397 & 0.376 & 250.3427 & 36.6378 & -248.3 & 2 & $\ldots$ \\
\hline 2185 & 53532 & 487 & 18.081 & 0.009 & 0.293 & 0.363 & 0.400 & 250.3905 & 36.5915 & -242.1 & 2 & $\ldots$ \\
\hline 2185 & 53532 & 488 & 19.313 & 0.018 & 0.294 & 0.449 & 0.422 & 250.3803 & 36.6619 & -239.6 & 2 & $\ldots$ \\
\hline 2185 & 53532 & 489 & 18.848 & 0.013 & 0.241 & 0.360 & 0.468 & 250.3864 & 36.7119 & -244.6 & 2 & $\ldots$ \\
\hline 2185 & 53532 & 490 & 19.105 & 0.013 & 0.292 & 0.389 & 0.377 & 250.4196 & 36.5921 & -242.2 & 2 & $\ldots$ \\
\hline 2185 & 53532 & 492 & 17.916 & 0.011 & 0.308 & 0.423 & 0.405 & 250.3583 & 36.6074 & -240.5 & 2 & $\ldots$ \\
\hline 2185 & 53532 & 494 & 18.957 & 0.014 & 0.310 & 0.375 & 0.348 & 250.4334 & 36.6197 & -243.8 & 2 & $\ldots$ \\
\hline 2185 & 53532 & 495 & 18.462 & 0.012 & 0.253 & 0.357 & 0.376 & 250.3132 & 36.6427 & -242.4 & 2 & $\ldots$ \\
\hline 2185 & 53532 & 496 & 19.600 & 0.017 & 0.355 & 0.473 & 0.395 & 250.3284 & 36.6039 & -245.6 & 2 & $\ldots$ \\
\hline 2185 & 53532 & 504 & 18.561 & 0.004 & 0.264 & 0.345 & 0.360 & 250.5587 & 36.6806 & -250.2 & 1 & $\ldots$ \\
\hline 2185 & 53532 & 507 & 18.897 & 0.016 & 0.324 & 0.402 & 0.443 & 250.4724 & 36.6777 & -243.3 & 2 & $\ldots$ \\
\hline 2185 & 53532 & 508 & 18.818 & 0.008 & 0.286 & 0.380 & 0.433 & 250.4435 & 36.7130 & -249.1 & 1 & $\ldots$ \\
\hline 2185 & 53532 & 516 & 19.603 & 0.020 & 0.321 & 0.485 & 0.545 & 250.4559 & 36.6134 & -239.8 & 2 & $\ldots$ \\
\hline 2185 & 53532 & 545 & 18.872 & 0.003 & 0.290 & 0.422 & 0.398 & 250.6272 & 36.7145 & -242.1 & 1 & $\ldots$ \\
\hline 2255 & 53565 & 116 & 15.802 & 0.002 & 0.536 & 0.765 & 0.881 & 250.5545 & 36.2678 & -249.5 & 1 & $\ldots$ \\
\hline 2255 & 53565 & 118 & 14.445 & 0.004 & 0.618 & 0.893 & 1.037 & 250.5464 & 36.3062 & -247.3 & 1 & 99 \\
\hline 2255 & 53565 & 144 & 15.112 & 0.004 & 0.586 & 0.822 & 0.957 & 250.4085 & 36.3039 & -246.9 & 1 & 99 \\
\hline 2255 & 53565 & 147 & 15.412 & 0.004 & -0.254 & -0.440 & -0.548 & 250.4520 & 36.3018 & -249.1 & 1 & 99 \\
\hline 2255 & 53565 & 152 & 12.840 & 0.001 & 0.847 & 1.200 & 1.414 & 250.4111 & 36.3777 & -249.9 & 1 & 99 \\
\hline 2255 & 53565 & 153 & 15.171 & 0.004 & 0.572 & 0.820 & 0.933 & 250.4041 & 36.3515 & -249.1 & 1 & 99 \\
\hline 2255 & 53565 & 156 & 15.511 & 0.004 & -0.286 & -0.468 & -0.588 & 250.4906 & 36.3635 & -244.8 & 1 & 98 \\
\hline 2255 & 53565 & 157 & 14.510 & 0.002 & 0.631 & 0.892 & 1.057 & 250.4289 & 36.3301 & -245.4 & 1 & 99 \\
\hline 2255 & 53565 & 160 & 14.601 & 0.022 & 0.595 & 0.878 & 1.044 & 250.4894 & 36.3321 & -249.9 & 1 & 99 \\
\hline 2255 & 53565 & 167 & 14.042 & 0.002 & 0.676 & 0.965 & 1.128 & 250.2349 & 36.3718 & -246.6 & 1 & 99 \\
\hline 2255 & 53565 & 172 & 14.493 & 0.004 & 0.614 & 0.880 & 0.972 & 250.3615 & 36.3904 & -248.9 & 1 & 99 \\
\hline 2255 & 53565 & 174 & 15.175 & 0.003 & -0.199 & -0.340 & -0.437 & 250.3136 & 36.3878 & -246.1 & 1 & 99 \\
\hline 2255 & 53565 & 175 & 15.232 & 0.002 & 0.577 & 0.824 & 0.919 & 250.3261 & 36.3471 & -242.6 & 1 & 99 \\
\hline 2255 & 53565 & 178 & 13.710 & 0.002 & 0.721 & 1.019 & 1.206 & 250.4017 & 36.2855 & -242.2 & 1 & 99 \\
\hline 2255 & 53565 & 426 & 15.024 & 0.001 & 0.571 & 0.831 & 0.989 & 250.3146 & 36.5174 & -244.4 & 1 & 99 \\
\hline 2255 & 53565 & 428 & 14.245 & 0.007 & 0.672 & 0.957 & 1.123 & 250.2309 & 36.5953 & -250.2 & 1 & 99 \\
\hline 2255 & 53565 & 431 & 13.412 & 0.021 & 0.728 & 1.065 & 1.255 & 250.3326 & 36.4106 & -250.0 & 1 & 99 \\
\hline 2255 & 53565 & 433 & 15.370 & 0.005 & -0.263 & -0.450 & -0.555 & 250.2911 & 36.5694 & -241.9 & 1 & 99 \\
\hline 2255 & 53565 & 436 & 14.753 & 0.003 & 0.619 & 0.885 & 1.026 & 250.3078 & 36.4174 & -241.8 & 1 & 99 \\
\hline 2255 & 53565 & 440 & 14.721 & 0.003 & 0.616 & 0.874 & 1.023 & 250.2608 & 36.4377 & -247.5 & 1 & 99 \\
\hline 2255 & 53565 & 464 & 13.338 & 0.003 & 0.703 & 1.005 & 1.178 & 250.3784 & 36.5035 & -244.4 & 1 & 99 \\
\hline 2255 & 53565 & 465 & 15.230 & 0.004 & -0.209 & -0.366 & -0.484 & 250.4422 & 36.4292 & -245.7 & 1 & 99 \\
\hline 2255 & 53565 & 468 & 15.375 & 0.008 & 0.538 & 0.810 & 0.891 & 250.3910 & 36.4529 & -242.3 & 1 & 98 \\
\hline 2255 & 53565 & 472 & 14.576 & 0.002 & 0.621 & 0.888 & 1.037 & 250.3964 & 36.4008 & -241.8 & 1 & 99 \\
\hline 2255 & 53565 & 474 & 13.116 & 0.003 & 0.797 & 1.132 & 1.301 & 250.3681 & 36.4510 & -243.0 & 1 & 98 \\
\hline 2255 & 53565 & 475 & 13.420 & 0.002 & 0.753 & 1.087 & 1.280 & 250.3134 & 36.4899 & -244.4 & 1 & 99 \\
\hline 2255 & 53565 & 477 & 13.740 & 0.002 & 0.697 & 0.997 & 1.154 & 250.3787 & 36.4254 & -249.1 & 1 & 99 \\
\hline 2255 & 53565 & 482 & 15.116 & 0.002 & -0.187 & -0.328 & -0.421 & 250.3339 & 36.6145 & -250.0 & 1 & 99 \\
\hline 2255 & 53565 & 486 & 14.665 & 0.001 & 0.611 & 0.886 & 1.050 & 250.3160 & 36.5549 & -243.1 & 1 & 99 \\
\hline 2255 & 53565 & 487 & 12.527 & 0.009 & 0.917 & 1.283 & 1.512 & 250.4381 & 36.4702 & -239.1 & 1 & 99 \\
\hline 2255 & 53565 & 490 & 15.825 & 0.003 & 0.517 & 0.755 & 0.889 & 250.3626 & 36.5661 & -244.7 & 1 & $\ldots$ \\
\hline 2255 & 53565 & 491 & 11.564 & 0.002 & 1.277 & 1.791 & 2.111 & 250.4595 & 36.4042 & -247.7 & 1 & 99 \\
\hline 2255 & 53565 & 493 & 13.683 & 0.013 & 0.722 & 1.035 & 1.204 & 250.4420 & 36.4546 & -248.3 & 1 & 99 \\
\hline 2255 & 53565 & 496 & 14.258 & 0.007 & 0.649 & 0.941 & 1.100 & 250.3886 & 36.5412 & -248.9 & 1 & 99 \\
\hline 2255 & 53565 & 498 & 11.446 & 0.014 & 1.352 & 1.938 & 2.281 & 250.4247 & 36.4476 & -247.8 & 1 & 99 \\
\hline 2255 & 53565 & 499 & 13.324 & 0.001 & 0.771 & 1.090 & 1.290 & 250.4365 & 36.3909 & -243.3 & 1 & 99 \\
\hline 2255 & 53565 & 501 & 13.201 & 0.004 & 0.783 & 1.128 & 1.314 & 250.4386 & 36.5185 & -243.5 & 1 & 99 \\
\hline 2255 & 53565 & 503 & 13.964 & 0.002 & 0.687 & 0.993 & 1.145 & 250.4857 & 36.5007 & -246.4 & 1 & 99 \\
\hline 2255 & 53565 & 504 & 13.826 & 0.004 & 0.705 & 1.019 & 1.167 & 250.4200 & 36.5698 & -248.5 & 1 & 99 \\
\hline
\end{tabular}


Table 4

(Continued)

\begin{tabular}{|c|c|c|c|c|c|c|c|c|c|c|c|c|}
\hline Plate & MJD & Fiber & $\begin{array}{c}r \\
(\mathrm{mag})\end{array}$ & $\begin{array}{l}\text { Error } \\
\text { (mag) }\end{array}$ & $\begin{array}{c}g-r \\
(\mathrm{mag})\end{array}$ & $\begin{array}{c}g-i \\
(\mathrm{mag})\end{array}$ & $\begin{array}{c}g-z \\
(\mathrm{mag})\end{array}$ & $\begin{array}{l}\text { R.A. } \\
(2000)\end{array}$ & $\begin{array}{l}\text { Decl. } \\
(2000)\end{array}$ & $\begin{array}{l}\text { Velocity } \\
\left(\mathrm{km} \mathrm{s}^{-1}\right)\end{array}$ & $\begin{array}{l}\text { Phot. } \\
\text { ref. }\end{array}$ & $\begin{array}{c}\text { PM prob } \\
(\%)\end{array}$ \\
\hline 2255 & 53565 & 505 & 15.367 & 0.003 & -0.231 & -0.388 & -0.488 & 250.4430 & 36.5538 & -246.8 & 1 & 99 \\
\hline 2255 & 53565 & 510 & 15.115 & 0.001 & 0.595 & 0.867 & 0.999 & 250.4508 & 36.5948 & -248.2 & 1 & 99 \\
\hline 2255 & 53565 & 517 & 13.955 & 0.010 & 0.687 & 1.002 & 1.124 & 250.4654 & 36.4590 & -241.8 & 1 & 99 \\
\hline 2255 & 53565 & 519 & 14.040 & 0.002 & 0.680 & 0.970 & 1.129 & 250.5015 & 36.4235 & -243.7 & 1 & 99 \\
\hline 2255 & 53565 & 520 & 14.373 & 0.004 & 0.644 & 0.930 & 1.042 & 250.4717 & 36.4231 & -241.2 & 1 & 99 \\
\hline 2255 & 53565 & 547 & 14.579 & 0.003 & 0.633 & 0.910 & 1.043 & 250.5786 & 36.5043 & -249.4 & 1 & 99 \\
\hline 2255 & 53565 & 548 & 14.836 & 0.002 & 0.629 & 0.887 & 1.037 & 250.5392 & 36.5664 & -245.2 & 1 & 99 \\
\hline 2255 & 53565 & 549 & 14.523 & 0.005 & 0.637 & 0.908 & 1.051 & 250.5105 & 36.5424 & -249.2 & 1 & 99 \\
\hline 2255 & 53565 & 550 & 15.197 & 0.004 & 0.589 & 0.842 & 0.956 & 250.5207 & 36.5268 & -246.5 & 1 & 99 \\
\hline 2255 & 53565 & 551 & 14.674 & 0.004 & 0.620 & 0.886 & 1.020 & 250.6084 & 36.4513 & -243.3 & 1 & 99 \\
\hline 2255 & 53565 & 552 & 13.934 & 0.002 & 0.689 & 0.988 & 1.149 & 250.5688 & 36.4162 & -241.4 & 1 & 99 \\
\hline 2255 & 53565 & 500 & 14.532 & 0.005 & 0.621 & 0.913 & 1.029 & 250.3637 & 36.5395 & -240.6 & 2 & 99 \\
\hline
\end{tabular}

References. 1. Clem et al. (2008), 2. An et al. (2008).

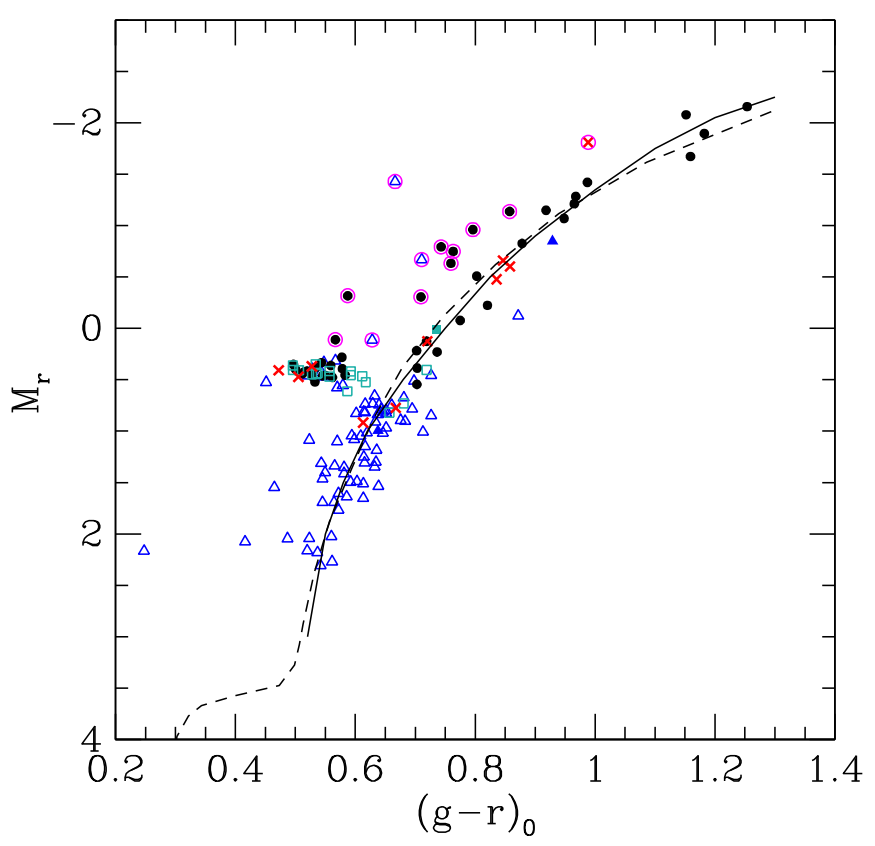

Figure 3. CMD of the globular cluster M71, using data from Clem et al. (2008) transformed to $g r$ using the transformations of Tucker et al. (2006). Only stars with more than a $70 \%$ probability of proper motion membership are plotted. Solid symbols denote stars where variable reddenings have been estimated and applied, while open symbols use $E(B-V)=0.28$. Black circles show stars with multiple radial velocity observations that are radial velocity members without variable velocities. Red crosses show stars with radial velocity variability suggesting that the star may be part of a binary system or a long-period variable. Blue/green squares indicate stars with one radial velocity measurement which are velocity members. Likely AGB stars are marked with a large magenta circle. Blue triangles denote stars without radial velocity measurements. The solid black line traces our new M71 giant branch fiducial, and the dotted line the Clem et al. (2008) fiducial.
(2013). We chose a radial velocity range of -251 to $-239 \mathrm{~km} \mathrm{~s}^{-1}$ to select radial velocity members for this cluster. Proper motions are available for all of the stars on the giant branch above the horizontal branch.

Data on individual cluster members are given in Table 4.

\section{3. $M 71$}

Unlike the two globular clusters previously discussed, M71 is a disk globular cluster in a low-latitude field with variable reddening (see, e.g., Casagrande et al. 2010). However, it is one of the few clusters in this metallicity range accessible from the north. Its low galactic latitude makes membership decisions more difficult because of the large number of foreground disk stars. In addition, M71's radial velocity is closer to that of the field stars because of its disk-like orbit: the difference is only $\sim-20 \mathrm{~km} \mathrm{~s}^{-1}$, compared to the M92 and M13 radial velocities which are $100 \mathrm{~km} \mathrm{~s}^{-1}$ (or more) different from the field star radial velocities.

Comparison of the giant branch fiducial of Clem et al. (2008) for M71 with other cluster fiducials suggests that the shape of the Clem et al. (2008) fiducial is slightly incorrect, presumably because of the larger probability of field star contamination on M71's giant branch, and because even genuine members have variable reddening and thus they do not trace a tight fiducial. We have therefore constructed a new fiducial for the M71 giant branch in $g$ and $r$, using only stars which are likely members, and have tightened up the CMD by estimating the variable reddening across M71's field, using the position of the main sequence turnoff in Clem's accurate photometry.

To construct the CMD for M71 using likely members only, we started with stars which had more than a $70 \%$ probability of membership from the proper motions of Cudworth (1985) and from unpublished data kindly made available by Kyle Cudworth 
Table 5

Variable Reddening Values (in $E(B-V)$ ) for M71

\begin{tabular}{|c|c|c|c|c|c|c|c|c|c|c|}
\hline \multirow{2}{*}{$\begin{array}{l}\text { Decl. Offset } \\
\text { (arcseconds) }\end{array}$} & \multicolumn{10}{|c|}{ R.A. Offset (arcseconds) } \\
\hline & -225 & -175 & -125 & -75 & -25 & 25 & 75 & 125 & 175 & 225 \\
\hline-225 & 0.03 & 0.02 & 0.00 & -0.01 & -0.01 & 0.00 & 0.00 & 0.00 & 0.00 & 0.03 \\
\hline-125 & 0.02 & 0.03 & 0.08 & 0.02 & -0.02 & -0.02 & -0.01 & 0.00 & 0.01 & 0.03 \\
\hline-75 & 0.03 & 0.02 & 0.02 & -0.02 & -0.02 & -0.02 & -0.02 & 0.00 & 0.02 & 0.02 \\
\hline-25 & 0.03 & 0.03 & 0.03 & -0.02 & 0.00 & 0.00 & 0.00 & 0.00 & 0.02 & 0.01 \\
\hline 25 & 0.04 & 0.04 & 0.02 & 0.01 & 0.01 & 0.00 & 0.00 & 0.01 & 0.00 & 0.00 \\
\hline 175 & 0.06 & 0.05 & 0.04 & 0.02 & 0.00 & 0.00 & 0.00 & 0.00 & 0.00 & 0.00 \\
\hline 225 & 0.07 & 0.06 & 0.05 & 0.05 & 0.02 & 0.00 & 0.00 & -0.01 & -0.01 & -0.01 \\
\hline
\end{tabular}

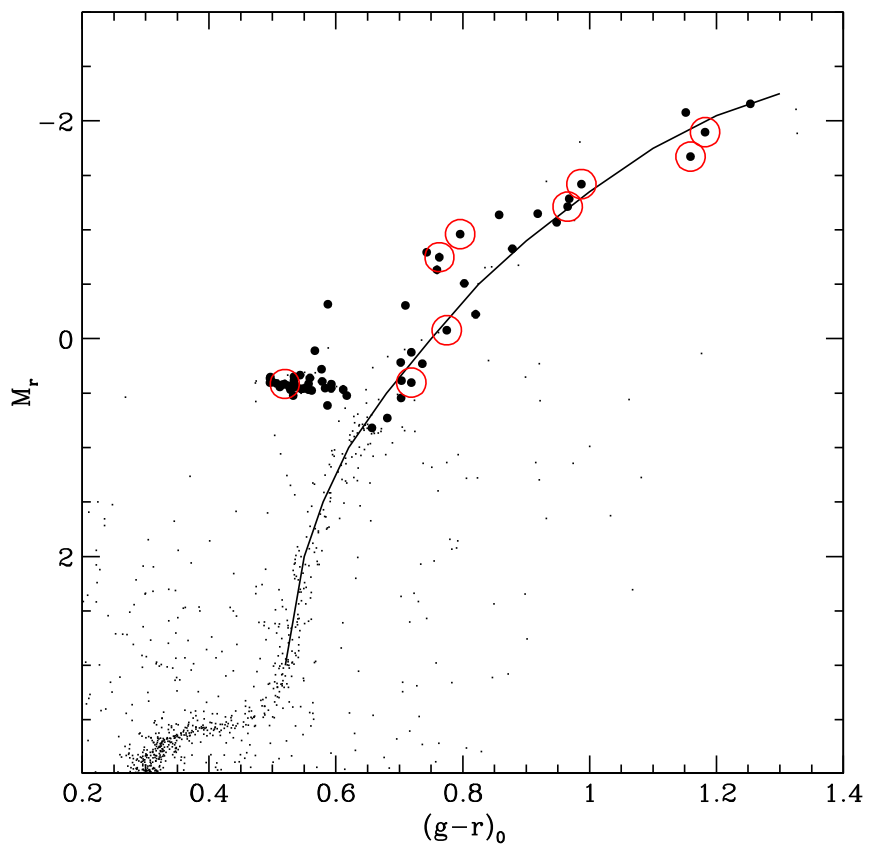

Figure 4. CMD of the globular cluster M71, using data from Clem et al. (2008). Stars without velocity data are shown as small black dots, while stars with proper motions and radial velocities indicating membership are shown as large black dots. Stars observed by SEGUE are shown by large red circles.

for the fainter stars. These data reach more than a magnitude below the horizontal branch, so are ideal for our purposes. We use the photometry of Clem et al. (2008), converted to gr using the transformations in Tucker et al. (2006).

For radial velocity membership data, in light of the small difference between M71's velocity and that of contaminating field stars, a more accurate velocity catalog was extremely helpful: T. Pryor (2015, private communication) kindly provided unpublished velocity data for almost all stars on or above the horizontal branch in M71. These data were obtained with high-resolution spectrographs on the DAO 48-inch and the KPNO $4 \mathrm{~m}$ and have errors $\leqslant 1 \mathrm{~km} \mathrm{~s}^{-1}$. There were also multiple observations for many of the stars, allowing likely binaries to be flagged via their radial velocity variability. The higher velocity accuracy allowed us to use a narrower window to define velocity membership: -20 to $-27 \mathrm{~km} \mathrm{~s}^{-1}$. We also rejected one star with both radial velocity and proper motion suggesting membership (star 1-1, on plate/MJD/fiber 2333/

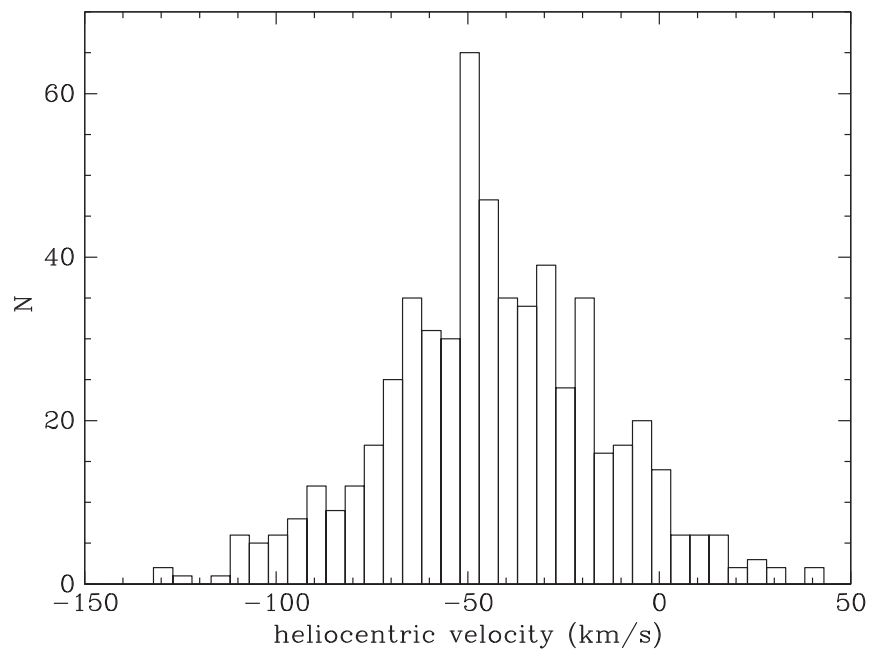

Figure 5. Velocity histogram of stars on the NGC 7789 plate. The broad spread of velocities from foreground/background disk stars is clear. All our targeted members are in the highest peak at $-50 \mathrm{~km} \mathrm{~s}^{-1}$, but roughly half the stars in this bin are still likely to be field stars.

$53682 / 165)$ but with radial velocity variations which may be due to binarity.

To estimate variable reddening values across the field of M71, we used the M71 photometry of Clem et al. (2008) to map the position of the main sequence turnoff across the field, using $g-i$ to provide a more sensitive measurement. We divided the region near M71 into square regions of size 50 arcsec, plotted the CMD near the turnoff for each region, and then overlaid the Clem et al. (2008) M71 fiducial, varying the reddening offset by eye until we obtained the best fit. The scatter around the fitted fiducial gives an estimate of the remaining variation in reddening inside the 50 arcmin square field (because the Clem et al. photometry was internally accurate to significantly better than $0.01 \mathrm{mag}$. at these bright magnitudes). This scatter had a range of $0.020-0.075$ in $g-i$ around the fiducial (equivalent to up to 0.05 in $E(g-r)$ ). Our reddening offsets are given in Table 5 and vary between +0.07 and -0.03 in $E(B-V)$ over the M71 field, which is 9.2 arcmin on a side.

Applying these reddening offsets to the confirmed cluster members (and using the global reddening and distance modulus values given in Table 1) results in the CMD shown in Figure 3, which can be compared with Figure 12 of Clem et al. (2008). (We used Clem's photometry in this case because the stars on 


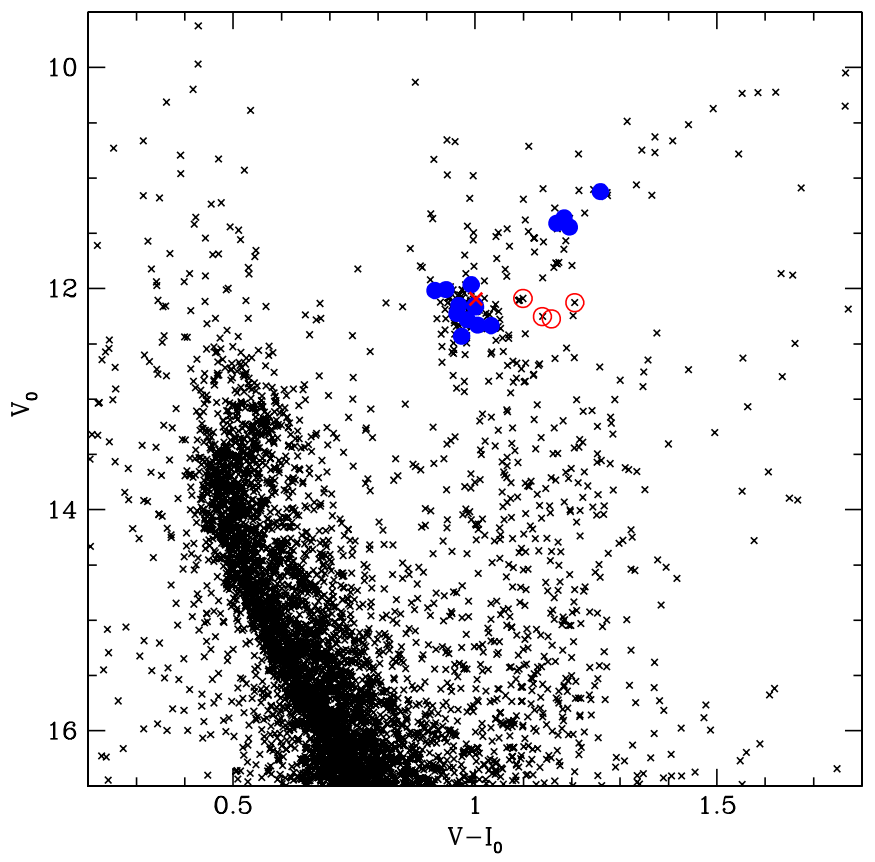

Figure 6. VI CMD of the open cluster NGC 7789, based on data from Gim et al. (1998b). Stars which are proper motion, radial velocity (from SEGUE) and CMD members are shown as filled blue circles. Stars which are proper motion and radial velocity members which we chose to reject because of their position on the CMD are shown with red open circles, while the star which Gim et al. (1998a) note has radial velocity variations is shown with a red cross.

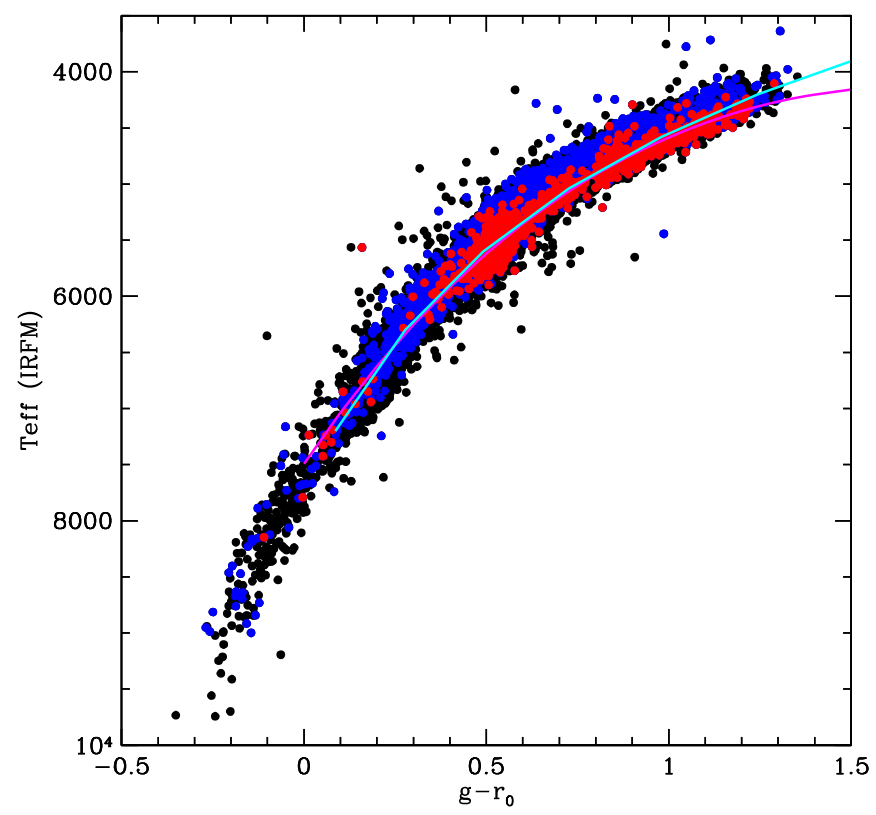

Figure 7. $(g-r)_{0}$ vs. $T_{\text {eff }}$ calculated using the IRFM for stars with good ugriz photometry and good 2 MASS colors. Black points show all stars, while blue points show stars with $E(B-V)<0.025$, and red points show the data used to obtain the transformation between $T_{\text {eff }}$ and $(g-r)_{0}$ for stars near solar metallicity, which have the additional restriction of $-0.2<[\mathrm{Fe} / \mathrm{H}]<0.25$ and $(g-r)_{0}>0$. Fit lines for Equations (1) and (2) are shown with cyan and magenta lines respectively.

M71's giant branch were either saturated or close to saturated in the original SDSS exposure.) Our CMD is significantly cleaner, with foreground stars removed and the red giant branch, red horizontal branch and asymptotic giant branch

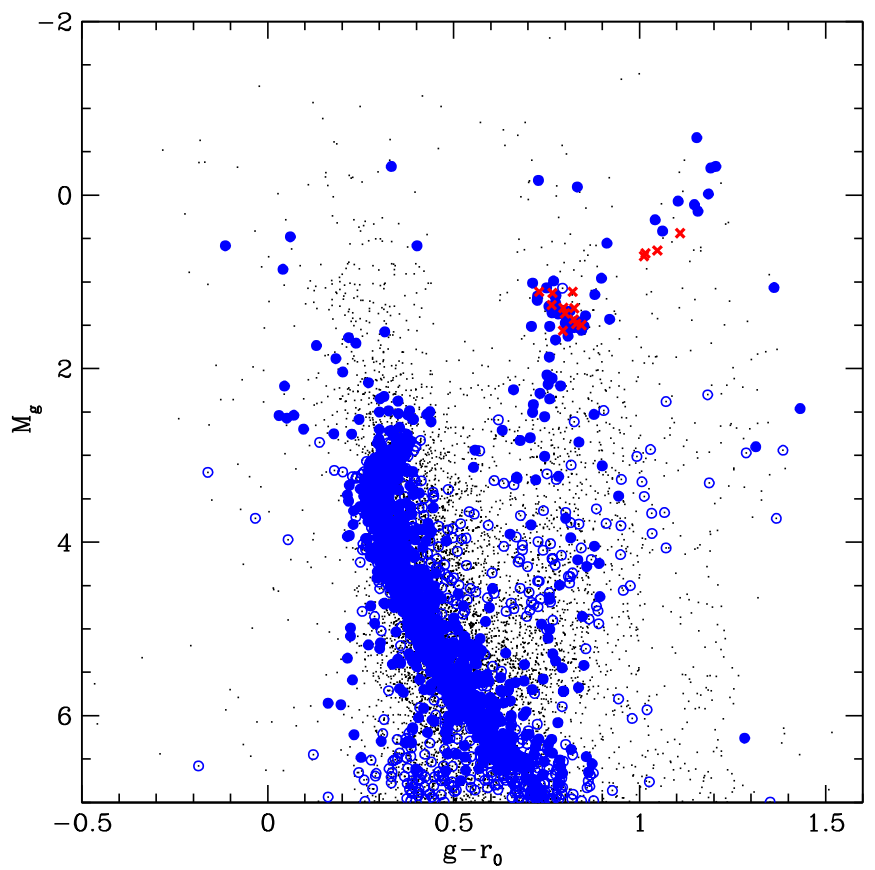

Figure 8. CMD of NGC 6819, a near "twin" of NGC 7789, with photometry from Platais et al. (2013). Small black points show all stars in the field, open blue circles are stars with proper motion membership probability (from Platais et al. 2013) greater than 50\%, and closed blue circles stars with membership probabilities greater than $80 \%$. Red crosses show our NGC 7789 members. The close agreement in clump star colors indicates that our transformation from $V I$ to $g r$ for NGC 7789 stars is quite accurate.

more clearly visible. Likely AGB stars are circled in magenta in the Figure: there are 11 of them. We show our improved fiducial for the M71 giant branch in the Figure. This fiducial is tabulated in Xue et al. (2014).

Table 6 lists our cluster members for M71. We also list the unpublished velocities of Pryor and collaborators, our adopted reddening offsets in $E(B-V)$ for each of these stars, and the ID of each star from Cudworth (1985) in order to make comparison with other studies easier. Figure 4 shows the confirmed members that were observed by SEGUE on M71's CMD. We have chosen to show two CMDs for M71 because showing the stars with spectroscopic observations in Figure 3 would detract from the membership information and the new fiducial presented there.

\subsection{NCG 7789}

NGC 7789 is a populous open cluster with a metal abundance slightly less than solar (Tautvaišienè et al. 2005) and age around 2 Gyr (Gim et al. 1998b). We selected targets using proper motion information from McNamara \& Solomon (1981) and velocities from Gim et al. (1998a). Our selected members have SEGUE radial velocities between -51.5 and $-48.2 \mathrm{~km} \mathrm{~s}^{-1}$. Figure 5 shows the radial velocities of stars in the SEGUE plate which observed NGC 7789, and illustrates some of the problems of obtaining reliable member lists for open clusters. All of the stars we targeted as likely giants are in the largest peak in the histogram, centered on $-50 \mathrm{~km} \mathrm{~s}^{-1}$, but the contribution of foreground and background disk stars to the velocity histogram, even at the exact cluster velocity, is significant. 
Table 6

M71 Members Observed by SEGUE

\begin{tabular}{|c|c|c|c|c|c|c|c|c|c|c|c|c|c|}
\hline Plate & MJD & Fiber & $\begin{array}{c}r \\
(\mathrm{mag})\end{array}$ & $\begin{array}{r}\text { Error } \\
(\mathrm{mag})\end{array}$ & $\begin{array}{c}g-r \\
(\mathrm{mag})\end{array}$ & $\begin{array}{r}\text { Error } \\
(\mathrm{mag})\end{array}$ & $\begin{array}{c}E(B-V) \\
\text { offset }\end{array}$ & $\begin{array}{l}\text { R.A. } \\
(2000)\end{array}$ & $\begin{array}{c}\text { Decl. } \\
(2000)\end{array}$ & $\begin{array}{l}\text { Velocity } \\
\left(\mathrm{km} \mathrm{s}^{-1}\right)\end{array}$ & $\begin{array}{c}\text { Error } \\
\left(\mathrm{km} \mathrm{s}^{-1}\right)\end{array}$ & $\begin{array}{c}\text { PM prob } \\
(\%)\end{array}$ & ID \\
\hline 2333 & 53682 & 163 & 11.766 & $\ldots$ & 1.480 & $\ldots$ & -0.01 & 298.4511 & 18.8007 & -20.5 & 0.2 & 97 & $1-45$ \\
\hline 2333 & 53682 & 167 & 14.106 & 0.002 & 0.828 & 0.004 & 0.00 & 298.4630 & 18.7697 & -26.2 & 0.6 & 85 & $1-19$ \\
\hline 2333 & 53682 & 173 & 12.477 & 0.002 & 1.273 & 0.004 & 0.00 & 298.4610 & 18.8189 & -24.9 & 0.3 & 97 & $1-53$ \\
\hline 2333 & 53682 & 185 & 12.886 & $\ldots$ & 1.051 & $\ldots$ & -0.02 & 298.4210 & 18.7683 & -23.2 & 0.5 & 96 & $1-95$ \\
\hline 2333 & 53682 & 191 & 14.203 & 0.004 & 1.069 & 0.005 & 0.04 & 298.4241 & 18.8108 & -25.3 & 0.8 & 95 & $1-59$ \\
\hline 2333 & 53682 & 224 & 13.695 & 0.004 & 1.114 & 0.008 & 0.03 & 298.3947 & 18.7727 & -23.4 & 0.4 & 95 & KC-39 \\
\hline 2333 & 53682 & 225 & 12.407 & 0.003 & 1.347 & 0.004 & 0.05 & 298.4062 & 18.7500 & -26.2 & 0.3 & 94 & A9 \\
\hline 2333 & 53682 & 239 & 12.072 & $\ldots$ & 1.488 & $\ldots$ & 0.02 & 298.4066 & 18.7914 & -26.7 & 0.3 & 90 & $1-77$ \\
\hline 2338 & 53679 & 150 & 12.729 & 0.004 & 1.104 & 0.004 & 0.00 & 298.4511 & 18.8071 & -21.6 & 0.5 & 97 & $1-56$ \\
\hline
\end{tabular}

Table 7

NGC 7789 Members Observed by SEGUE

\begin{tabular}{|c|c|c|c|c|c|c|c|c|c|}
\hline Plate & MJD & Fiber & $\begin{array}{c}V \\
(\mathrm{mag})\end{array}$ & $\begin{array}{c}V-I \\
(\mathrm{mag})\end{array}$ & $\begin{array}{c}(g-r)_{0} \\
(\text { transf) }\end{array}$ & $\begin{array}{l}\text { R.A. } \\
(2000)\end{array}$ & $\begin{array}{c}\text { Decl. } \\
(2000)\end{array}$ & $E(B-V)$ & $\begin{array}{l}\text { Velocity } \\
\left(\mathrm{km} \mathrm{s}^{-1}\right)\end{array}$ \\
\hline 2377 & 53991 & 151 & 12.794 & 1.336 & 0.820 & 359.5293 & 56.6800 & 0.25 & -49.1 \\
\hline 2377 & 53991 & 162 & 12.839 & 1.284 & 0.765 & 359.3542 & 56.6434 & 0.25 & -46.7 \\
\hline 2377 & 53991 & 175 & 12.913 & 1.288 & 0.730 & 359.3507 & 56.6600 & 0.27 & -50.6 \\
\hline 2377 & 53991 & 176 & 13.055 & 1.306 & 0.800 & 359.3866 & 56.6721 & 0.25 & -48.0 \\
\hline 2377 & 53991 & 178 & 12.962 & 1.331 & 0.795 & 359.3378 & 56.5841 & 0.24 & -47.6 \\
\hline 2377 & 53991 & 191 & 12.981 & 1.310 & 0.763 & 359.2386 & 56.6153 & 0.25 & -44.4 \\
\hline 2377 & 53991 & 200 & 12.273 & 1.539 & 1.012 & 359.2176 & 56.5608 & 0.25 & -44.6 \\
\hline 2377 & 53991 & 232 & 12.188 & 1.528 & 1.048 & 358.9569 & 56.6550 & 0.25 & -48.1 \\
\hline 2377 & 53991 & 439 & 13.260 & 1.316 & 0.794 & 359.1707 & 56.6964 & 0.25 & -46.8 \\
\hline 2377 & 53991 & 461 & 12.305 & 1.540 & 1.016 & 359.2311 & 56.7525 & 0.27 & -46.2 \\
\hline 2377 & 53991 & 489 & 12.982 & 1.344 & 0.824 & 359.2382 & 56.6971 & 0.25 & -50.7 \\
\hline 2377 & 53991 & 493 & 13.111 & 1.328 & 0.823 & 359.2648 & 56.7225 & 0.25 & -47.5 \\
\hline 2377 & 53991 & 494 & 13.160 & 1.349 & 0.829 & 359.1855 & 56.7149 & 0.25 & -47.6 \\
\hline 2377 & 53991 & 506 & 11.986 & 1.617 & 1.109 & 359.5007 & 56.8368 & 0.26 & -47.1 \\
\hline 2377 & 53991 & 515 & 13.164 & 1.377 & 0.844 & 359.4409 & 56.8448 & 0.25 & -47.6 \\
\hline
\end{tabular}

To produce our membership list, we rejected four of the stars which were both proper motion and velocity members because of their position in the CMD shown in Figure 6. While these stars may be members whose variable reddening moves them away from the cluster sequence, we have chosen to be conservative and reject them, since a major aim of this paper is simply to produce a collection of cluster stars which have a high likelihood of being members. We also rejected one star because (Gim et al. 1998a) noted that it had radial velocity variations.

Since there are no ugriz data available for NGC 7789, we show $V$ and $I$ photometry from Gim et al. (1998b) in the CMD of Figure 6, with the cluster members observed by SEGUE highlighted. The large contribution from foreground/background disk stars is clear in the CMD as well. The variable reddening can be seen in the scatter of colors and magnitudes in the clump in particular. Individual estimates of reddening values were available for about one-third of the giants from the Vilnius photometry of Bartašiute \& Tautvaišienè (2004). We used these values where available, and their cluster value $(E(B-$ $V)=0.25$ ) otherwise.

Since there are no ugriz or $u^{\prime} g^{\prime} r^{\prime} i^{\prime} z^{\prime}$ data available for this cluster, we transformed from $V-K_{s}$ to $g-r$ via $T_{\text {eff }}$ measurements. First, we used the relation between $V-K_{s}$ and $T_{\text {eff }}$ of Ramírez \& Meléndez (2005), the $V$ magnitudes of Gim et al. (1998b), 2MASS $K_{s}$ magnitudes and $E(B-V)$ to derive $T_{\text {eff }}$ for each star. We chose to use $V-K_{S}$ because its relation between effective temperature and color is the least sensitive to $[\mathrm{Fe} / \mathrm{H}]$ and gravity (Bessell 2008). We then derived a relation between $T_{\text {eff }}$ and $g-r$ using the Infrared Flux Method (IRFM hereafter) colors of stars observed with SEGUE which had near-solar abundances. We selected 2068 stars with SDSS spectra, good ugriz, 2MASS $J, H$ and $K_{s}$ colors (Skrutskie et al. 2006), SSPP metallicities between -0.2 and +0.25 (which have a mean metallicity of -0.05 , the same as NGC 7789) and low reddening: $E(B-V)$ from Schlegel et al. (1998) less than 0.025. L. Casagrande et al. (in preparation) computed individual Infrared Flux Method (see Blackwell \& Shallis 1977; Casagrande et al. 2010) temperatures for a large number of stars observed by SEGUE, including these stars. Figure 7 shows the relationship between $(g-r)_{0}$ and this IRFM $T_{\text {eff }}$ for all stars with low reddening (blue points) and for stars with near solar abundance (red points). We fitted cubic relationships to these low-reddening, near solar abundance stars as follows, first defining $Q=5040 / T_{\text {eff. }}$.

$$
\begin{aligned}
Q= & 0.6728+0.4265(g-r)_{0} \\
& +0.08841(g-r)_{0}^{2}-0.08881(g-r)_{0}{ }^{3} .
\end{aligned}
$$

The differences between this line and the actual $T_{\text {eff }}$ values have a sigma of $112 \mathrm{~K}$. 


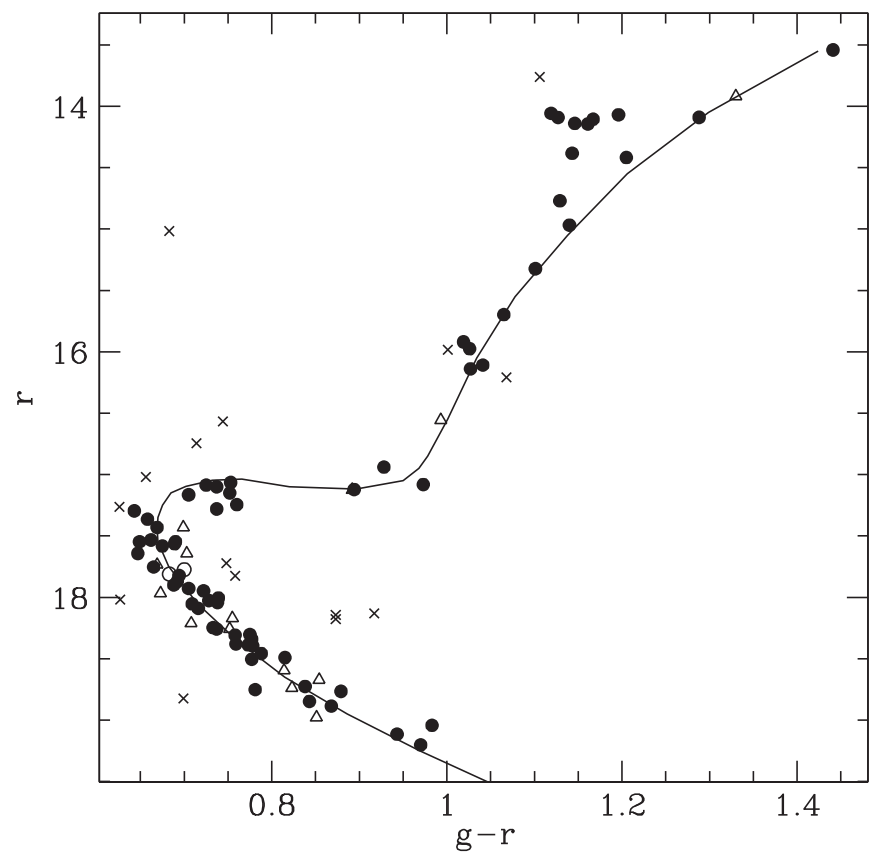

Figure 9. $g r$ CMD of the rich open cluster NGC 6791, using data from An et al. (2008). Circles and triangles show radial velocity members. Stars with proper motion membership probabilities higher than $70 \%$ are shown as filled circles, while those with proper motion membership probabilities smaller than $70 \%$ are shown with open triangles. If no proper motion is available for the star, it is shown with an open circle. Crosses show stars classified as nonmembers because of their position in the CMD.

The inverse relation is:

$$
(g-r)_{0}=-0.3672-0.7188 Q+2.281 Q^{2}-0.4688 Q^{3} .
$$

The differences between the fit line and the actual $(g-r)_{0}$ values is $0.047 \mathrm{mag}$. Assuming errors of $0.02 \mathrm{mag}$. for the $V$ and $K_{s}$ magnitudes gives a random error estimate of $0.055 \mathrm{mag}$. for this entire process. Lastly, we added $100 \mathrm{~K}$ to the $T_{\text {eff }}$ from Ramírez \& Meléndez (2005) to correct for the difference in absolute scale between their IRFM scale and that used by Casagrande et al. (see Casagrande et al. 2010, for a detailed discussion of this point) then used Equation (2) to calculate the value of $(g-r)_{0}$ for each star.

We checked this transformation using an open cluster which has a similar age and metallicity to NGC 7789 but also has $g^{\prime} r^{\prime}$ observations (Platais et al. 2013), NGC 6819. We used the standard transformations of Tucker et al. (2006) to transform the NGC 6819 photometry into $g$ and $r$, and the cluster reddening of $E(B-V)=0.16$ (Anthony-Twarog et al. 2014), to make the CMD shown in Figure 8. Our 15 confirmed NGC 7789 red giants and clump stars are overplotted. The solid blue symbols are proper motion members of NGC 6819, while the red crosses are our NGC 7789 members. Since there are a range of cluster distance moduli for NGC 6819 in the literature, we adjusted the NGC 6819 absolute magnitudes until the clumps of the two clusters coincided. The agreement of $g-r$ color between the two clumps is gratifying, suggesting that our transformation from $V I$ to $g-r$ is quite accurate.

Data on NGC 7789 members are listed in Table 7. The $V$ and I photometry are from Gim et al. (1998b), radial velocities from our SEGUE data and reddening values from Bartašiute \& Tautvaišienè (2004), and $(g-r)_{0}$ values from the transformation described above.

\subsection{NGC 6791}

NGC 6791 is a particularly useful cluster because it anchors our calibrations at the metal-rich end, having $[\mathrm{Fe} / \mathrm{H}]=+0.39$. We used run 5416 of the photometry from An et al. (2008) and applied the "ubercalibration" corrections given in Table 1 of An et al. (2013). To determine membership we used proper motion data from K. Cudworth (2015, private communication), choosing all stars with proper motion membership probability greater than $70 \%$, velocities between -60 and $-48 \mathrm{~km} \mathrm{~s}^{-1}$, and finally, by removing several stars whose position on the CMD was not consistent with membership of the cluster. We remind the reader that our aim here is to obtain a collection of the most likely cluster members, not a complete set, and such rejection is a conservative step for this purpose. Figure 9 shows the NGC 6791 stars observed by SEGUE on the cluster CMD, and Table 8 lists the likely members of the cluster.

\section{A CLUSTER WITH NO PROPER-MOTION DATA: BERKELEY 29}

Useful proper motion data are not available for the open cluster Be 29. However, the cluster was observed using the SDSS imager on the $2.5 \mathrm{~m}$ telescope at the end of the SDSS-III imaging season in 2009 January (Aihara et al. 2011), and we include its $g r$ photometry in this work. Following the procedure in An et al. 2008 used for the other SDSS clusters, we reduced SDSS imaging frames using the DAOPHOT/ALLFRAME suite of programs (Stetson 1987; 1994), and converted DAOPHOT magnitudes into the SDSS asinh values (Lupton et al. 1999) using photometric zero points, extinction coefficients, and airmass values. We tied our cluster photometry to the ubercal system, as for the other cluster photometry discussed in Section 3, by deriving zero-point offsets between DAOPHOT and DR8 photometry in low density fields near Be 29. We checked the adjusted DAOPHOT magnitudes in these cluster flanking fields with more recent values in DR12 (Alam et al. 2015), and found 0.008 mag differences in each of the $g$ and $r$ passbands. The $g r$ CMD of Be 29 is shown in Figure 10: the red clump can be seen near $g-r=0.8$ and $r=17$.

Be 29's stars were in general fainter than those in the other clusters we studied, because we aimed to obtain unsaturated photometry of its giant branch. This led to larger than average velocity errors on the Be 29 stars. Because Be 29's radial velocity is close to that of the disk stars in the field, contamination from foreground/background disk stars is a problem, and we do not have a very accurate radial velocity to use for member identification. However, as we showed in our discussion of NGC 7789, even full velocity and proper motion data for each star is not sufficient to identify members for open clusters. Our strategy, therefore, was to adopt a wide velocity window (10-40 $\mathrm{km} \mathrm{s}^{-1}$ ) and visually inspect the spectra of the stars within this window to determine if they were giants (and thus likely to belong to Be 29) or dwarfs (and so not cluster members given their colors and magnitudes). The criteria we used for this visual inspection included the strengths of the $\mathrm{Mg} b / \mathrm{H}$ feature near $5170 \AA$, the strengths of the $\mathrm{Ca}$ I line at $4227 \AA$ (both described in detail in Morrison et al. 2003), and the relative strength of the $\mathrm{Sr}$ II line at $4077 \AA$ to the nearby Fe I lines at 4045 and $4063 \AA$ (Rose 1984).

Table 9 provides detail on the Be 29 members observed by SEGUE. 
Table 8

NGC 6791 Members Observed by SEGUE

\begin{tabular}{|c|c|c|c|c|c|c|c|c|c|c|c|}
\hline Plate & MJD & Fiber & $\begin{array}{c}r \\
(\mathrm{mag})\end{array}$ & $\begin{array}{l}\text { Error } \\
(\mathrm{mag})\end{array}$ & $\begin{array}{c}g-r \\
(\mathrm{mag})\end{array}$ & $\begin{array}{r}g-i \\
(\mathrm{mag})\end{array}$ & $\begin{array}{c}g-z \\
(\mathrm{mag})\end{array}$ & $\begin{array}{l}\text { R.A. } \\
\text { (2000) }\end{array}$ & $\begin{array}{l}\text { Decl. } \\
\text { (2000) }\end{array}$ & $\begin{array}{l}\text { Velocity } \\
\left(\mathrm{km} \mathrm{s}^{-1}\right)\end{array}$ & $\begin{array}{c}\text { PM prob } \\
(\%)\end{array}$ \\
\hline 2800 & 54326 & 151 & 16.943 & 0.005 & 0.927 & 1.235 & 1.439 & 290.3105 & 37.7758 & -47.6 & 88 \\
\hline 2800 & 54326 & 152 & 17.904 & 0.011 & 0.687 & 0.915 & 1.070 & 290.2779 & 37.8023 & -45.8 & 96 \\
\hline 2800 & 54326 & 154 & 15.327 & 0.006 & 1.100 & 1.495 & 1.745 & 290.2560 & 37.8014 & -47.1 & 90 \\
\hline 2800 & 54326 & 156 & 16.112 & 0.005 & 1.040 & 1.395 & 1.639 & 290.2894 & 37.7840 & -43.9 & 75 \\
\hline 2800 & 54326 & 159 & 14.061 & 0.005 & 1.118 & 1.528 & 1.762 & 290.2762 & 37.7499 & -45.8 & 99 \\
\hline 2800 & 54326 & 160 & 15.923 & 0.004 & 1.018 & 1.369 & 1.577 & 290.3084 & 37.7526 & -47.2 & 85 \\
\hline 2800 & 54326 & 161 & 17.126 & 0.007 & 0.893 & 1.195 & 1.361 & 290.2689 & 37.7212 & -46.5 & 97 \\
\hline 2800 & 54326 & 167 & 13.923 & 0.007 & 1.329 & $\ldots$ & 2.178 & 290.2547 & 37.7037 & -47.7 & 68 \\
\hline 2800 & 54326 & 169 & 14.148 & 0.007 & 1.160 & 1.583 & 1.820 & 290.2448 & 37.7203 & -45.3 & 77 \\
\hline 2800 & 54326 & 170 & 13.545 & 0.006 & 1.440 & & 2.484 & 290.2191 & 37.7412 & -47.9 & 92 \\
\hline 2800 & 54326 & 172 & 17.284 & 0.006 & 0.736 & 0.955 & 1.086 & 290.2085 & 37.7977 & -45.3 & 79 \\
\hline 2800 & 54326 & 173 & 17.085 & 0.007 & 0.972 & 1.314 & 1.497 & 290.2308 & 37.7971 & -47.9 & 81 \\
\hline 2800 & 54326 & 174 & 14.073 & 0.005 & 1.195 & 1.625 & 1.895 & 290.2536 & 37.7594 & -46.8 & 97 \\
\hline 2800 & 54326 & 175 & 14.109 & 0.005 & 1.166 & 1.572 & 1.836 & 290.2536 & 37.7777 & -49.5 & 97 \\
\hline 2800 & 54326 & 180 & 14.095 & 0.004 & 1.126 & 1.520 & 1.767 & 290.2203 & 37.7592 & -45.5 & 93 \\
\hline 2800 & 54326 & 181 & 14.421 & 0.003 & 1.204 & 1.656 & 1.926 & 290.1882 & 37.7428 & -49.6 & 98 \\
\hline 2800 & 54326 & 182 & 17.435 & 0.007 & 0.668 & 0.893 & 0.968 & 290.1303 & 37.7752 & -47.3 & 98 \\
\hline 2800 & 54326 & 183 & 14.143 & 0.006 & 1.145 & 1.541 & 1.812 & 290.1889 & 37.7883 & -52.4 & 88 \\
\hline 2800 & 54326 & 184 & 17.368 & 0.009 & 0.657 & 0.878 & 0.979 & 290.1277 & 37.7548 & -50.6 & 98 \\
\hline 2800 & 54326 & 185 & 14.387 & 0.005 & 1.142 & 1.527 & 1.775 & 290.1635 & 37.7437 & -46.8 & 98 \\
\hline 2800 & 54326 & 187 & 17.154 & 0.007 & 0.751 & 0.998 & 1.146 & 290.1433 & 37.8011 & -47.0 & 73 \\
\hline 2800 & 54326 & 188 & 17.932 & 0.007 & 0.704 & 0.933 & 1.052 & 290.1961 & 37.7612 & -45.8 & 91 \\
\hline 2800 & 54326 & 189 & 16.560 & 0.009 & 0.992 & 1.324 & 1.518 & 290.1688 & 37.7852 & -45.9 & 55 \\
\hline 2800 & 54326 & 190 & 15.701 & 0.006 & 1.064 & 1.439 & 1.672 & 290.1767 & 37.7642 & -46.3 & 88 \\
\hline 2800 & 54326 & 194 & 17.300 & 0.011 & 0.642 & 0.852 & 0.968 & 290.1742 & 37.7060 & -52.4 & 77 \\
\hline 2800 & 54326 & 197 & 15.979 & 0.007 & 1.025 & 1.377 & 1.606 & 290.1808 & 37.7214 & -46.7 & 92 \\
\hline 2800 & 54326 & 199 & 14.094 & 0.004 & 1.287 & $\ldots$ & 2.107 & 290.1639 & 37.8013 & -45.7 & 94 \\
\hline 2800 & 54326 & 431 & 17.103 & 0.005 & 0.736 & 0.993 & 1.109 & 290.1247 & 37.8115 & -40.9 & 98 \\
\hline 2800 & 54326 & 465 & 14.972 & 0.004 & 1.139 & 1.570 & 1.844 & 290.2405 & 37.8170 & -44.9 & 85 \\
\hline 2800 & 54326 & 475 & 14.774 & 0.010 & 1.128 & 1.540 & 1.823 & 290.1927 & 37.8196 & -43.2 & 99 \\
\hline 2800 & 54326 & 479 & 17.874 & 0.008 & 0.691 & 0.947 & 1.064 & 290.1633 & 37.8346 & -46.8 & 95 \\
\hline 2800 & 54326 & 480 & 17.249 & 0.007 & 0.759 & 1.039 & 1.180 & 290.1579 & 37.8190 & -44.4 & 82 \\
\hline 2821 & 54393 & 141 & 17.951 & 0.008 & 0.721 & 0.946 & 1.066 & 290.2928 & 37.7322 & -46.8 & 89 \\
\hline 2821 & 54393 & 142 & 18.703 & 0.010 & 0.839 & 1.086 & 1.255 & 290.2954 & 37.7891 & -40.9 & 96 \\
\hline 2821 & 54393 & 145 & 17.970 & 0.008 & 0.672 & 0.911 & 0.956 & 290.3149 & 37.7871 & -44.0 & 68 \\
\hline 2821 & 54393 & 146 & 18.216 & 0.009 & 0.707 & 0.936 & 1.065 & 290.2860 & 37.7175 & -43.9 & 68 \\
\hline 2821 & 54393 & 149 & 17.586 & 0.006 & 0.674 & 0.878 & 0.966 & 290.2926 & 37.7521 & -43.2 & 70 \\
\hline 2821 & 54393 & 161 & 17.537 & 0.008 & 0.661 & 0.865 & 0.976 & 290.2675 & 37.7325 & -48.9 & 83 \\
\hline 2821 & 54393 & 165 & 18.742 & 0.012 & 0.822 & 1.119 & 1.234 & 290.1696 & 37.7074 & -48.6 & 67 \\
\hline 2821 & 54393 & 166 & 18.509 & 0.010 & 0.776 & 1.027 & 1.210 & 290.2165 & 37.7927 & -43.8 & 85 \\
\hline 2821 & 54393 & 167 & 18.392 & 0.013 & 0.772 & 1.037 & 1.095 & 290.2531 & 37.7614 & -43.8 & 91 \\
\hline 2821 & 54393 & 169 & 18.398 & 0.013 & 0.777 & 1.042 & 1.203 & 290.2357 & 37.7495 & -43.6 & 94 \\
\hline 2821 & 54393 & 172 & 18.174 & 0.011 & 0.754 & 0.991 & 1.120 & 290.2331 & 37.7795 & -48.4 & 69 \\
\hline 2821 & 54393 & 173 & 18.094 & 0.013 & 0.715 & 0.941 & 1.043 & 290.2340 & 37.7255 & -46.5 & 76 \\
\hline 2821 & 54393 & 174 & 18.263 & 0.014 & 0.736 & 0.934 & 1.075 & 290.2744 & 37.7682 & -46.9 & 80 \\
\hline 2821 & 54393 & 176 & 18.983 & 0.014 & 0.850 & 1.154 & 1.265 & 290.2389 & 37.7979 & -42.3 & 63 \\
\hline 2821 & 54393 & 177 & 18.312 & 0.011 & 0.757 & 1.000 & 1.151 & 290.2552 & 37.7811 & -44.8 & 92 \\
\hline 2821 & 54393 & 178 & 18.462 & 0.011 & 0.787 & 1.051 & 1.215 & 290.2708 & 37.7936 & -47.7 & 84 \\
\hline 2821 & 54393 & 179 & 17.434 & 0.013 & 0.698 & 0.925 & 1.037 & 290.2332 & 37.6950 & -46.3 & 61 \\
\hline 2821 & 54393 & 182 & 18.009 & 0.008 & 0.738 & 0.964 & 1.044 & 290.1917 & 37.7502 & -44.9 & 98 \\
\hline 2821 & 54393 & 183 & 17.738 & 0.009 & 0.668 & 0.870 & 0.983 & 290.1618 & 37.7224 & -48.2 & 68 \\
\hline 2821 & 54393 & 187 & 17.646 & 0.009 & 0.646 & 0.868 & 0.972 & 290.1851 & 37.7333 & -49.2 & 72 \\
\hline 2821 & 54393 & 188 & 18.770 & 0.010 & 0.878 & 1.157 & 1.295 & 290.1615 & 37.7461 & -42.9 & 72 \\
\hline 2821 & 54393 & 190 & 19.206 & 0.018 & 0.969 & 1.265 & 1.434 & 290.2118 & 37.7134 & -43.0 & 85 \\
\hline 2821 & 54393 & 191 & 18.251 & 0.011 & 0.732 & 0.980 & 1.100 & 290.1620 & 37.7770 & -50.4 & 95 \\
\hline 2821 & 54393 & 193 & 18.495 & 0.009 & 0.814 & 1.069 & 1.168 & 290.1486 & 37.7595 & -44.2 & 97 \\
\hline 2821 & 54393 & 194 & 18.047 & 0.009 & 0.737 & 0.971 & 1.087 & 290.1838 & 37.7774 & -45.6 & 95 \\
\hline 2821 & 54393 & 195 & 18.852 & 0.012 & 0.842 & 1.122 & 1.293 & 290.2029 & 37.7670 & -44.5 & 89 \\
\hline 2821 & 54393 & 196 & 18.058 & 0.012 & 0.708 & 0.939 & 1.101 & 290.2135 & 37.7412 & -48.2 & 97 \\
\hline 2821 & 54393 & 197 & 17.757 & 0.006 & 0.664 & 0.888 & 0.988 & 290.1443 & 37.7864 & -47.3 & 94 \\
\hline 2821 & 54393 & 198 & 18.678 & 0.012 & 0.819 & 1.105 & 1.209 & 290.1754 & 37.7626 & -48.5 & 68 \\
\hline 2821 & 54393 & 199 & 18.890 & 0.014 & 0.867 & 1.150 & 1.331 & 290.1903 & 37.7149 & -48.0 & 72 \\
\hline
\end{tabular}


Table 8

(Continued)

\begin{tabular}{lccccccccccc}
\hline \hline Plate & MJD & Fiber & $\begin{array}{c}r \\
(\mathrm{mag})\end{array}$ & $\begin{array}{c}\text { Error } \\
(\mathrm{mag})\end{array}$ & $\begin{array}{c}g-r \\
(\mathrm{mag})\end{array}$ & $\begin{array}{c}g-i \\
(\mathrm{mag})\end{array}$ & $\begin{array}{c}g-z \\
(\mathrm{mag})\end{array}$ & $\begin{array}{c}\text { R.A. } \\
(2000)\end{array}$ & $\begin{array}{c}\text { Decl. } \\
(2000)\end{array}$ & $\begin{array}{c}\text { Velocity } \\
(\mathrm{km} \mathrm{s})^{-1}\end{array}$ & $\begin{array}{c}\text { PM prob } \\
(\%)\end{array}$ \\
\hline 2821 & 54393 & 200 & 18.260 & 0.011 & 0.751 & 0.974 & 1.090 & 290.1816 & 37.7957 & -47.5 & 56 \\
2821 & 54393 & 232 & 19.101 & 0.016 & 0.954 & 1.342 & 1.515 & 290.1246 & 37.7310 & -46.9 & 87 \\
2821 & 54393 & 235 & 17.550 & 0.009 & 0.689 & 0.900 & 0.991 & 290.1257 & 37.7643 & -42.1 & 95 \\
2821 & 54393 & 436 & 18.307 & 0.008 & 0.774 & 1.082 & 1.253 & 290.1259 & 37.8133 & -44.3 & 91 \\
2821 & 54393 & 439 & 17.568 & 0.006 & 0.688 & 0.926 & 1.027 & 290.1193 & 37.7980 & -44.1 \\
\hline
\end{tabular}

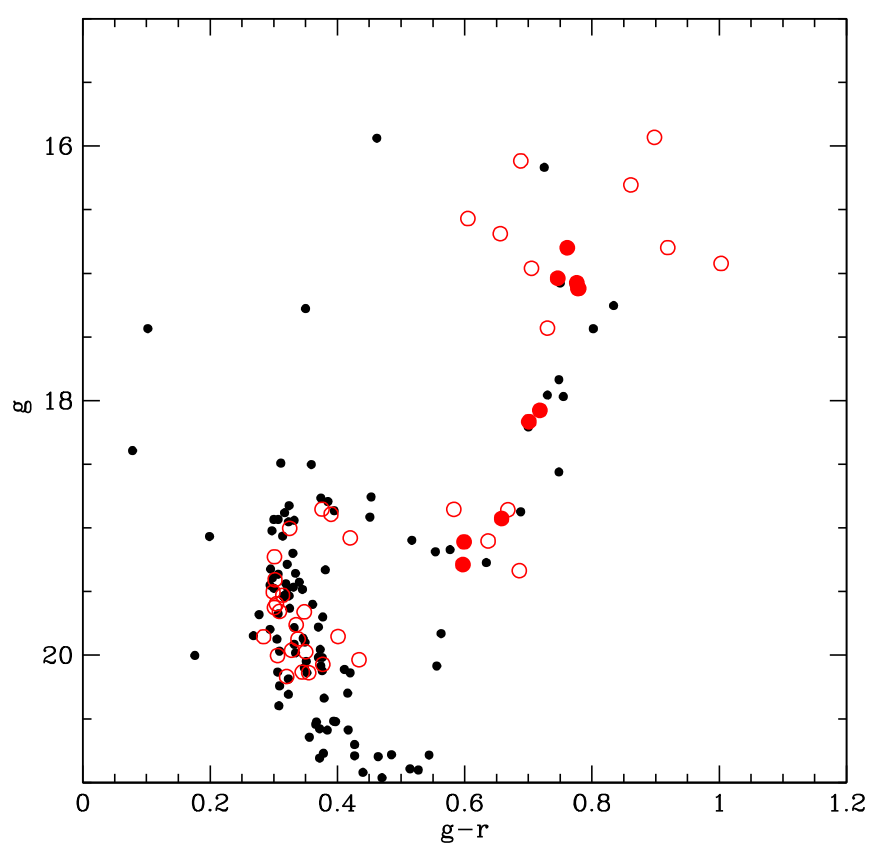

Figure 10. CMD of the open cluster Be 29. Filled black circles show stars from the DAOphot reductions of the SDSS photometry which are within 3 arcmin radius of the cluster center. The most luminous giants in this plot are on the red horizontal branch. Open red circles show radial velocity members in the giant branch color range, while filled red circles show stars confirmed spectroscopically to be giants. It can be seen that there is significant contamination by non-cluster members in the CMD.

\section{SUMMARY}

We present CMDs and membership information for stars from the globular clusters M92, M13 and M71 and the open clusters Berkeley 29, NGC 7789 and NGC 6791.

For the nearby globular clusters M92 and M13 ([Fe/ $\mathrm{H}]=-2.4$ and -1.6 respectively), we describe the procedures used to observe the brightest giants spectroscopically on the SDSS $2.5 \mathrm{~m}$ and the transformation of the $u^{\prime} g^{\prime} r^{\prime} i^{\prime} z^{\prime}$ magnitudes of these bright stars to ugriz. We used multiple criteria to isolate cluster members: the SEGUE radial velocity, proper motions and CMD position. We tabulate griz magnitudes and SEGUE radial velocity measures for the 79 M92 members and the 146 M13 members.

In the disk globular cluster M71 $([\mathrm{Fe} / \mathrm{H}]=-0.8)$, reddening is variable due to its low galactic latitude. We mapped this variation using the position of the main sequence turnoff in the photometry of Clem et al. (2008) and give a table of variable reddening values for this cluster. The improved reddening estimates produced a much cleaner CMD and allowed us identify possible AGB members and to construct an improved fiducial for the red giant branch, important for our $\mathrm{K}$ giant distance estimates. This fiducial is tabulated in Xue et al. (2014). We give $g r$ photometry, reddening offsets and velocities for the nine members on the giant or horizontal branch which were observed by SEGUE.

Open clusters are traditionally harder to study because their low galactic latitudes and low concentration lead to large contamination from non-members, even when accurate radial velocity and proper motions are available.

The open cluster $\mathrm{Be} 29([\mathrm{Fe} / \mathrm{H}]=-0.4)$ has no proper motion data available, and because its stars are relatively faint, the SEGUE radial velocities have errors of order $10 \mathrm{~km} \mathrm{~s}^{-1}$. Thus we supplemented the velocity and CMD position criteria with a visual inspection of the SEGUE spectra in order to cull out the foreground dwarf stars in the field of this cluster. We tabulate $g r$ photometry and velocity measurements for the 10 cluster members on the lower giant branch and red clump for this cluster.

The open cluster NGC $7789([\mathrm{Fe} / \mathrm{H}]=0.0)$ has both radial velocity and proper motion data available, but no ugriz or $u^{\prime} g^{\prime}$ $r^{\prime} i^{\prime} z^{\prime}$ data. We derived a transformation from $V-K_{s}$ to $g r$ for stars with near-solar abundances via recent $T_{\text {eff }}$ calibrations of stars with good SDSS photometry and SEGUE spectra. We validated this transformation via CMD comparisons with the very similar open cluster NGC 6819, and give photometry and velocities for the 15 giant branch or red clump members of NGC 7789 observed by SEGUE.

The super-metal-rich cluster NGC $6791([\mathrm{Fe} / \mathrm{H}]=+0.4)$ has higher concentration than many open clusters, so the combination of CMD position, radial velocity and proper motion is sufficient to identify cluster members. We tabulate griz photometry and SEGUE velocities for the 67 cluster members observed by SEGUE.

The information presented in this paper will be useful in calibrations and tests of the SEGUE observations, particularly for red giant stars, and will be used in the series of papers titled "The SEGUE K giant survey." These papers include a detailed description of the technique of identifying $\mathrm{K}$ giants in SEGUE data (H. Morrison et al. 2015, in preparation), the calculation of distances for the $\sim 6000 \mathrm{~K}$ giants identified by the survey (Xue et al. 2014), a study of substructure in halo giants out to $100 \mathrm{kpc}$ (Janesh et al. 2015), the study of the radial profile of the halo out to $60 \mathrm{kpc}$, which also includes an estimate of the (slight) radial metallicity gradient in the halo (Xue et al. 2015) and two papers currently in preparation, one on the halo MDF and its variation with distance, and the other on the $[\alpha / \mathrm{Fe}]$ ratios of $\sim 2000 \mathrm{~K}$ giants in the halo. 
Table 9

Berkeley 29 Members Observed by SEGUE

\begin{tabular}{|c|c|c|c|c|c|c|c|c|c|c|c|}
\hline Plate & MJD & Fiber & $\begin{array}{c}g \\
(\mathrm{mag})\end{array}$ & $\begin{array}{l}\text { Error } \\
\text { (mag) }\end{array}$ & $\begin{array}{c}g-r \\
(\mathrm{mag})\end{array}$ & $\begin{array}{l}\text { Error } \\
\text { (mag) }\end{array}$ & $\begin{array}{l}\text { R.A. } \\
(2000)\end{array}$ & $\begin{array}{c}\text { Decl. } \\
(2000)\end{array}$ & $\begin{array}{l}\text { Velocity } \\
\left(\mathrm{km} \mathrm{s}^{-1}\right)\end{array}$ & $\begin{array}{c}\text { Error } \\
\left(\mathrm{km} \mathrm{s}^{-1}\right)\end{array}$ & Other ID $^{\mathrm{a}}$ \\
\hline 3335 & 54922 & 113 & 19.110 & 0.015 & 0.599 & 103.3311 & 16.8730 & 0.017 & 26.57 & 2.22 & $\ldots$ \\
\hline 3335 & 54922 & 195 & 16.799 & 0.007 & 0.761 & 103.1608 & 16.8460 & 0.011 & 34.56 & 1.04 & $\ldots$ \\
\hline 3335 & 54922 & 462 & 18.926 & 0.011 & 0.658 & 103.2417 & 16.9463 & 0.018 & 39.85 & 1.85 & $\ldots$ \\
\hline 3335 & 54922 & 474 & 19.288 & 0.014 & 0.597 & 103.1888 & 16.8793 & 0.021 & 18.02 & 2.80 & $\ldots$ \\
\hline 3335 & 54922 & 481 & 17.039 & 0.006 & 0.746 & 103.2836 & 16.9279 & 0.008 & 17.01 & 0.86 & S398, C801, F948 \\
\hline 3335 & 54922 & 495 & 17.118 & 0.007 & 0.779 & 103.3010 & 16.9836 & 0.010 & 17.81 & 0.95 & F1437 \\
\hline 3335 & 54922 & 496 & 17.074 & 0.006 & 0.776 & 103.2311 & 16.9610 & 0.009 & 18.13 & 0.89 & S602 \\
\hline 3335 & 54922 & 497 & 17.120 & 0.008 & 0.778 & 103.2566 & 16.9392 & 0.010 & 18.22 & 0.85 & S159 \\
\hline 3335 & 54922 & 498 & 18.165 & 0.007 & 0.701 & 103.2626 & 16.9245 & 0.011 & 21.69 & 1.34 & $\ldots$ \\
\hline 3335 & 54922 & 508 & 18.076 & 0.011 & 0.718 & 103.2837 & 16.9631 & 0.015 & 16.68 & 1.53 & $\ldots$ \\
\hline
\end{tabular}

Note.

${ }^{a}$ Identifications are from S: Sestito et al. (2008), C: Carraro et al. (2004) and F: Frinchaboy et al. (2006).

We thank Tad Pryor for kindly sharing his unpublished velocity data for M71 with us, and Bruce Twarog for suggesting that we use NGC 6819 to help transform the NGC 7789 data to $g r$. We also thank the anonymous referee for a very helpful report. This research used the facilities of the Canadian Astronomy Data Centre operated by the National Research Council of Canada with the support of the Canadian Space Agency. This publication also makes use of data products from the Two Micron All Sky Survey, which is a joint project of the University of Massachusetts and the Infrared Processing and Analysis Center/California Institute of Technology, funded by the National Aeronautics and Space Administration and the National Science Foundation. This work was supported in part by the National Science Foundation under Grant No. PHYS-1066293 and the hospitality of the Aspen Center for Physics, and by grants AST-1009886 to H. L. M. and AST-121989 to H. L. M., P. H. and C.R. T. C. B. acknowledges partial support for this work by grant PHY 0822648: Physics Frontiers Center/Joint Institute for Nuclear Astrophysics (JINA), awarded by the U.S. National Science Foundation.

Funding for SDSS-III has been provided by the Alfred P. Sloan Foundation, the Participating Institutions, the National Science Foundation, and the U.S. Department of Energy Office of Science. The SDSS-III web site is http://www. sdss3.org/. SDSS-III is managed by the Astrophysical Research Consortium for the Participating Institutions of the SDSS-III Collaboration including the University of Arizona, the Brazilian Participation Group, Brookhaven National Laboratory, Carnegie Mellon University, University of Florida, the French Participation Group, the German Participation Group, Harvard University, the Instituto de Astrofisica de Canarias, the Michigan State/Notre Dame/ JINA Participation Group, Johns Hopkins University, Lawrence Berkeley National Laboratory, Max Planck Institute for Astrophysics, Max Planck Institute for Extraterrestrial Physics, New Mexico State University, New York University, Ohio State University, Pennsylvania State University, University of Portsmouth, Princeton University, the Spanish Participation Group, University of Tokyo, University of Utah, Vanderbilt University, University of Virginia, University of Washington, and Yale University.

\section{REFERENCES}

Aihara, H., Allende Prieto, C., An, D., et al. 2011, ApJS, 193, 29

Alam, S., Albareti, F. D., Allende Prieto, C., et al. 2015, ApJS, 219, 12

Allende Prieto, C., Sivarani, T., Beers, T. C., et al. 2008, AJ, 136, 2070

An, D., Beers, T. C., Johnson, J. A., et al. 2013, ApJ, 763, 65

An, D., Johnson, J. A., Clem, J. L., et al. 2008, ApJS, 179, 326

An, D., Terndrup, D. M., Pinsonneault, M. H., et al. 2007, ApJ, 655, 233

Anthony-Twarog, B. J., Deliyannis, C. P., \& Twarog, B. A. 2014, AJ, 148,51

Anthony-Twarog, B. J., Tanner, D., Cracraft, M., \& Twarog, B. A. 2006, AJ, 131, 461

Bartašiutè, S., \& Tautvaišienè, G. 2004, A\&AS, 294, 225

Bessell, M. S. 2008, MmSAI, 79, 317

Blackwell, D. E., \& Shallis, M. J. 1977, MNRAS, 180, 177

Bragaglia, A., \& Tosi, M. 2006, AJ, 131, 1544

Brogaard, K., Bruntt, H., Grundahl, F., et al. 2011, A\&A, 525, A2

Carraro, G., Bresolin, F., Villanova, S., et al. 2004, AJ, 128, 1676

Carraro, G., Villanova, S., Demarque, P., et al. 2006, ApJ, 643, 1151

Carretta, E., Gratton, R. G., Clementini, G., \& Fusi Pecci, F. 2000, ApJ, 533,215

Casagrande, L., Ramírez, I., Meléndez, J., Bessell, M., \& Asplund, M. 2010, A\&A, 512, A54

Clem, J. L., Vanden Berg, D. A., \& Stetson, P. B. 2008, AJ, 135, 682

Cudworth, K. M. 1985, AJ, 90, 65

Cudworth, K. M., \& Monet, D. G. 1979, AJ, 84, 774

Eisenstein, D. J., Weinberg, D. H., Agol, E., et al. 2011, AJ, 142, 72

Frinchaboy, P. M., Muñoz, R. R., Phelps, R. L., Majewski, S. R., \& Kunkel, W. E. 2006, AJ, 131, 922

Fukugita, M., Ichikawa, T., Gunn, J. E., et al. 1996, AJ, 111, 1748

Gim, M., Hesser, J. E., McClure, R. D., \& Stetson, P. B. 1998a, PASP, 110,1172

Gim, M., Vandenberg, D. A., Stetson, P. B., Hesser, J. E., \& Zurek, D. R. 1998b, PASP, 110, 1318

Gratton, R., Bragaglia, A., Carretta, E., \& Tosi, M. 2006, ApJ, 642, 462

Grundahl, F., Stetson, P. B., \& Andersen, M. I. 2002, A\&A, 395, 481

Gunn, J. E., Carr, M., Rockosi, C., et al. 1998, AJ, 116, 3040

Gunn, J. E., Siegmund, W. A., Mannery, E. J., et al. 2006, AJ, 131, 2332

Harris, W. E. 1996, AJ, 112, 1487

Jacobson, H. R., Pilachowski, C. A., \& Friel, E. D. 2011, AJ, 142, 59

Janesh, W., Morrison, H. L., Ma, Z., et al. 2015, ApJ, in press (arXiv:1503.09133)

Kraft, R. P., \& Ivans, I. I. 2003, PASP, 115, 143

Lee, Y. S., Beers, T. C., Sivarani, T., et al. 2008a, AJ, 136, 2022

Lee, Y. S., Beers, T. C., Sivarani, T., et al. 2008b, AJ, 136, 2050

Lupton, R. H., Gunn, J. E., \& Szalay, A. S. 1999, AJ, 118, 1406

McNamara, B. J., \& Solomon, S. 1981, A\&AS, 43, 337

Morrison, H. L., Norris, J., Mateo, M., et al. 2003, AJ, 125, 2502

Önehag, A., Gustafsson, B., \& Korn, A. 2014, A\&A, 562, A102

Padmanabhan, N., Schlegel, D. J., Finkbeiner, D. P., et al. 2008, ApJ, 674, 1217

Perryman, M. A. C., Lindegren, L., Kovalevsky, J., et al. 1997, A\&A, 323, L49 
Peterson, R. C., \& Green, E. M. 1998, ApJL, 502, L39

Platais, I., Gosnell, N. M., Meibom, S., et al. 2013, AJ, 146, 43

Ramírez, I., \& Meléndez, J. 2005, ApJ, 626, 465

Rees, R. F., Jr. 1992, AJ, 103, 1573

Rose, J. A. 1984, AJ, 89, 1238

Schlegel, D. J., Finkbeiner, D. P., \& Davis, M. 1998, ApJ, 500, 525

Sestito, P., Bragaglia, A., Randich, S., et al. 2008, A\&A, 488, 943

Skrutskie, M. F., Cutri, R. M., Stiening, R., et al. 2006, AJ, 131, 1163

Smee, S. A., Gunn, J. E., Uomoto, A., et al. 2013, AJ, 146, 32

Smith, J. A., Tucker, D. L., Kent, S., et al. 2002, AJ, 123, 212

Smolinski, J. P., Lee, Y. S., Beers, T. C., et al. 2011, AJ, 141, 89
Stetson, P. B. 1987, PASP, 99, 191

Stetson, P. B. 1994, PASP, 106, 250

Stoughton, C., Lupton, R. H., Bernardi, M., et al. 2002, AJ, 123, 485

Tautvaišienè, G., Edvardsson, B., Puzeras, E., \& Ilyin, I. 2005, A\&A, 431, 933

Taylor, B. J. 2007, AJ, 133, 370

Tucker, D. L., Kent, S., Richmond, M. W., et al. 2006, AN, 327, 821

Yanny, B., Rockosi, C., Newberg, H. J., et al. 2009, AJ, 137, 4377

York, D. G., Adelman, J., Anderson, J. E., Jr., et al. 2000, AJ, 120, 1579

Xue, X.-X., Ma, Z., Rix, H.-W., et al. 2014, ApJ, 784, 170

Xue, X.-X., Rix, H.-W., Ma, Z., et al. 2015, ApJ, 809, 144 